Universidad de Barcelona

ISSN I578-0007

Vol. XXIV. Núm. 245

I de agosto de 2020

DOI: https://doi.org/10.1344/ara2020.245.32II5

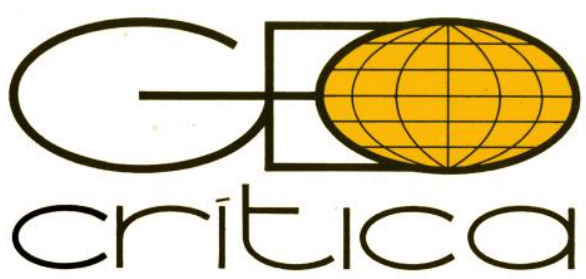

\title{
LA MONTAÑA EN LOS PROGRAMAS INTERNACIONALES DE LA UNESCO (1972-2002): DEL MAN AND BIOSPHERE AL AÑO INTERNACIONAL DE LAS MONTAÑAS. FUENTES PARA SU INVESTIGACIÓN
}

\author{
Pere Sunyer Martín \\ Departamento de Sociología. Universidad Autónoma Metropolitana-Iztapalapa \\ peresunyer@live.com \\ Dhttps://orcid.org/0000-0002-7383-2399
}

\begin{abstract}
La montaña en los programas internacionales de la UNESCO (1972-2002): del Man and Biosphere al Año Internacional de las Montañas. Fuentes para su investigación (Resumen)

Con la aprobación en junio de 1992 de la Agenda 21, en la Cumbre Mundial sobre el Medio Ambiente y el desarrollo en Río de Janeiro, se establecieron las bases para la actuación de los Estados en pos del desarrollo sostenible, en el siglo XXI. Uno de los ámbitos de trabajo eran las montañas, recogidas en su capítulo 13. Su inclusión fue resultado de un largo camino iniciado veinte años antes, en 1971, con el programa de la UNESCO Man and Biosphere (MAB) que culminó con la celebración del 2002 Año Internacional de las montañas; un camino que condujo a un grupo de entusiastas de estos espacios desde los estudios del medio físico al humano, y desde la necesidad de salvaguardar su diversidad física y biológica a la de proteger la diversidad cultural de los pueblos que en ella habitan. De ahí se crearon algunos programas y organismos cuyos trabajos y actividades son todavía referentes para aquellos que deseen acercarse a entender el funcionamiento y riqueza a nivel planetario de las montañas.
\end{abstract}

Palabras clave: ecosistemas de montaña; Man and Biosphere (MAB); Agenda de montaña; 2002 Año Internacional de las Montañas. 
The mountain in the UNESCO international programmes (1972-2002): from Man and Biosphere Programme to the International Year of Mountains. Sources for its research (Abstract)

On June 1992 the Agenda 21 was approved at the Rio de Janeiro World Summit on Environment and Development. Agenda 21 stablished the bases for the action of States towards sustainability during 21st Century. Mountain areas were one of the topics. Chapter 13 of the aforementioned document was the result of a long journey that started twenty years earlier, in 1971, with the UNESCO Man and Biosphere program (MAB) and culminated in 2002 International Mountain Year. A journey that led a mountain enthusiastic little group from studies of its physical and biological features to human geography, and from the need to safeguard their physical and biological diversity to that of protect the cultural diversity of peoples that inhabit it. Some programs, organisms and institutions were created and their works are still landmarks to those who wish to understanding the functioning and planetary wealthiness of mountains.

Key-Words: Mountain ecosystems, Man and Biosphere programme (MAB), Mountain Agenda, 2002 International Year of Mountains

Una consulta en la web sobre el tema "montaña" (en español) arroja un resultado de 159 millones de entradas; 220 millones con montagne, más de 4.000 millones con mountain. Un número considerable, como puede apreciarse. Si nos remitimos al ámbito académico encontramos 4,7 millones de entradas con "mountain" en Google Scholar"; medio millón con "montaña” y 656.000 con "montagne". Con un calificativo, por ejemplo "mountain geography", las entradas se reducen sensiblemente.

Una atenta revisión de las primeras páginas en la búsqueda general del término, aparte de mostrar la amplia variedad de montañas que hay en el mundo y su valor estético, nos acerca a su definición, sus características; nos informa también de cuáles son las más elevadas del planeta, entre otros muchos temas. También junto a estas primeras entradas hay una amplia gama de portales dedicados a la venta de productos para la práctica del montañismo, anuncios de clubes deportivos y guías, que nos hablan del sentido lúdico-deportivo que tiene para muchos la montaña: un espacio de ocio para ciertos sectores sociales del planeta. Paralelamente, unas pocas tratan de adentrarse en su estudio, profundizar en sus características biológicas, físicas o en los problemas que aquejan a las poblaciones que en ellas viven -un espacio de vida-. Una de ellas está dedicada a su día internacional, el 11 de diciembre.

Este día proclamado en 2003 por las Naciones Unidas nos ofrece otro panorama de las montañas. Nos habla de su relevancia mundial a pesar de que sólo ocupan aproximadamente una cuarta parte del planeta, de que abastecen de agua a una parte importante de la población mundial y son de las áreas más diversas en cuanto a su riqueza biológica, pero también étnica y culturalmente hablando ${ }^{2}$.

La consulta de esta página nos encamina a las celebraciones de 2002 Año internacional de las montañas y hacia las reflexiones que llevaron a incluir estos espacios terrestres entre los ecosistemas frágiles del mundo. En 1992, por primera vez en un documento de alcance internacional, se reconocía la importancia de las montañas y su necesaria protección por todos los

\footnotetext{
${ }^{1} 167.000$ en Scopus con "mountain".

${ }^{2}$ ONU, Organización de las Naciones Unidas, 2019.
} 
La montaña em los programas internacionales de la UNESCO (1972-2002): del Man and Biosphere...

valores que atesora. Así, en la Agenda o Programa 21, como también se conoce al informe final de la Conferencia Internacional sobre el Medio ambiente y el desarrollo de Río de Janeiro de 1992 o "Cumbre de la Tierra", se dedicaba uno de sus capítulos, el décimo tercero, exclusivamente a las montañas ${ }^{3}$. Su título era Managing fragile ecosystems: sustainable mountains development (Gestión de ecosistemas frágiles: desarrollo sostenible de las montañas).

En aquellos años, el presidente de la Comisión de las Naciones Unidas para el Medio ambiente y el Desarrollo, Maurice F. Strong, decía que "los ecosistemas frágiles de montaña son el ámbito de vida de numerosas poblaciones que en ellas viven y proporcionan numerosos servicios ambientales para muchísimas más personas. Se necesitan adoptar medidas de protección de forma urgente para las generaciones presentes y futuras" 4 .

Desde la Cumbre de la Tierra el número de portales relativos a organismos e instituciones de investigación en el ámbito de la montaña, a grupos de apoyo a proyectos de desarrollo, y a la comprensión de los espacios de montaña, en general, y de sus poblaciones se ha multiplicado de forma exponencial. Llegar a esta situación, sin embargo, fue resultado de un largo camino que tuvo un momento clave a mediados de los años sesenta precisamente en las investigaciones geográficas desarrolladas por Carl Troll desde un par de decenios antes y que tuvo en la aprobación del programa de la UNESCO Hombre y biosfera (Man and Biosphere, MAB) ${ }^{5}$ su principal valedor. Hoy se considera la montaña como un objeto de conocimiento complejo, en el que es necesario cada vez más desarrollar estudios de carácter interdisciplinario para lo cual dos especialidades parecen disputarse el campo, la geografía de la montaña y la montología 6 .

Inicialmente, el objetivo de este artículo era ocuparme de forma sistemática de los principales portales existentes en la actualidad dedicados a los espacios de montaña del mundo de manera que pudiera servir de guía a futuras investigaciones sobre el tema. Sin embargo, me ha parecido que no se entendería si antes no ofrecía un panorama amplio de cómo se fue desarrollando a nivel internacional la preocupación por estos espacios y a su vez ir presentando los programas, organismos e instituciones, así como algunas de las personalidades, que han ido impulsando los estudios de montaña en los tiempos recientes.

De esta manera, queremos aproximarnos a lo que creemos que es el origen del interés contemporáneo de los estudios de la montaña en el ámbito internacional, tomando como punto de partida el impulso dado desde la UNESCO bajo el programa Hombre y biosfera a principios de los años 70, la dirección que fue tomando hasta el año 1992, año de celebración de la Cumbre Mundial sobre el Medio ambiente y el desarrollo en Río de Janeiro, para finalizar aproximadamente en 2002

\footnotetext{
${ }^{3}$ ONU, 1992a.

${ }^{4}$ Strong. Citado en Environmental conservation, 1993, p. 96.

${ }^{5}$ Nota del autor: Usaré indistintamente el término de "Hombre y Biosfera", Man and Biosphere o más comúnmente $M A B$ para referirme a este programa de la UNESCO.

${ }^{6} \mathrm{Si}$ bien en un apartado final haremos referencia a la "geografía de la montaña", queda fuera del interés de este artículo abordar la larga aportación de la geografía al descubrimiento de la montaña y su conversión como objeto científico. En la bibliografía revisada para este trabajo, mayoritariamente anglosajona, hemos constatado la ignorancia existente con respecto la aportación de la geografía humana y regional francesa en los estudios de la montaña, heredera de una larga tradición geográfica. Esta ausencia de referencias se extiende a los documentos consultados de la Unión Geográfica Internacional (UGI o IGU, por sus siglas en inglés).
} 
con la celebración del Año Internacional de las Montañas. En esos treinta años de recorrido ha aparecido abundante material bibliográfico y documental, mucho del cual se haya todavía disponible en la web, reflejo de la preocupación que desde distintas especialidades de las ciencias naturales y sociales ha despertado la montaña, sobre todo desde la geografía ${ }^{7}$.

\section{Los problemas ecológicos de las montañas}

Hombre y biosfera $(M A B)$ es el primer gran programa de carácter ambiental que se establece a escala internacional. Entre sus objetivos estaban el de concienciar y planear el uso racional de los recursos de la biosfera y trabajar por su conservación ${ }^{8}$. Su aprobación en 1971 en la Asamblea General de las Naciones Unidas vino precedida de otras tantas reuniones en una de las cuales, la geografía y las montañas tuvieron una destacada presencia.

En efecto, algunos autores destacan el simposio de la UNESCO en la ciudad de México, los días 1 al 3 de agosto de 1966 dedicado a Geoecología de las regiones de montaña de la América tropical tras el cual estaba el geógrafo alemán Carl Troll quien llevaba muchos años dedicado al estudio de las montañas ${ }^{9}$. Fue este simposio, aseguran algunos autores, el que permitió que el asunto de la montaña y sus problemas formase parte de uno de los temas que se iban a abordar en Man and Biosphere.

El programa $M A B$ se organizó en grupos de trabajo (Working Groups en el original), la mayoría de ellos encargado de los principales ecosistemas considerados que, a su vez, eran equiparables a las grandes regiones naturales del globo (véase Cuadro 1$)^{10}$. Todos ellos ayudaron a reflexionar acerca de los problemas que atañían la relación ser humano y los recursos. El tema de las montañas en el Working Group $^{11}$ del proyecto número 6 no podía faltar.

\footnotetext{
${ }^{7}$ En un apartado final, ofrecemos algunos vínculos de internet relacionados con el estudio de las montañas de eventos, instituciones, actividades y principales publicaciones todavía vigentes.

${ }^{8}$ Preámbulo al documento 16 C/78 de la UNESCO (1970).

${ }^{9}$ Jack D. Ives (2012), un científico muy vinculado a los estudios sobre la montaña, y González Trueba (2012) son de esta opinión. Las comunicaciones fueron publicadas en 1968 bajo el título Proceedings of the 9th Pacific Science Congress on Mountain Geography. Physical and Human Dimensions, y ese mismo año bajo la autoría de Carl Troll (1968) con el título Geo-ecology of the Mountainous Regions of the Tropical Americas. Uno de sus primeros estudios fue Studien zur Vergleichunden Geographie der Hochgebirge der Erde (1941) [Estudios de geografía comparada de las altas montañas de la Tierra] a partir del enfoque de ecología del paisaje que posteriormente denominó "geoecología" (González Trueba, 2012, p. 175).

${ }^{10}$ Fueron inicialmente trece proyectos que regionalizaban en siete zonas el mundo según sus características naturales - grandes regiones naturales, climáticas o geomórfológicas-y a sus respectivas poblaciones. Los seis restantes se dedicaban a temas diversos, material genético, áreas urbanas e industriales, grandes obras de ingeniería, entre otros.

${ }^{11}$ Nota: En adelante, usaré indistintamente Grupo de trabajo o Working Group para referirme al equipo encargado del estudio de los ambientes de montaña del programa $M A B$.
} 
La montaña em los programas internacionales de la UNESCO (1972-2002): del Man and Biosphere...

Cuadro 1. Proyectos por grupo de trabajo del Programa Man and Biosphere (1971)

\begin{tabular}{|c|c|}
\hline$N^{o}$ Proyecto & Tema \\
\hline Proyecto 1 & Ecosistemas forestales tropicales y subtropicales \\
\hline Proyecto 2 & Paisajes forestales de climas templados y mediterráneos \\
\hline Proyecto 3 & Ecosistemas herbáceos: sabanas, pastos de zonas áridas y templadas, tundra \\
\hline Proyecto 4 & Ecosistemas de zonas áridas y semiáridas \\
\hline Proyecto 5 & Ecosistemas lacustres, marismas, ríos, deltas, estuarios y zonas costeras \\
\hline Proyecto 6 & Ecosistemas de montaña \\
\hline Proyecto 7 & Ecosistemas insulares \\
\hline Proyecto 8 & Conservación de áreas naturales y su material genético \\
\hline Proyecto 9 & Efectos en ecosistemas acuáticos de pesticidas y fertilizantes agrícolas \\
\hline Proyecto 10 & Efectos de las grandes obras de ingeniería \\
\hline Proyecto 11 & Efectos del uso de la energía en sistemas urbanos e industriales \\
\hline Proyecto 12 & Interacciones entre transformaciones ambientales y cambios genéticos y demográficos \\
\hline Proyecto 13 & Percepción de la calidad ambiental \\
\hline
\end{tabular}

Fuente: Elaboración personal a partir de UNESCO, 1972 (SC/MD/26).

\section{Las reuniones del Working Group núm.6 y los rasgos principales de las montañas}

El Grupo de trabajo núm. 6 llevaba por título Impacto de las actividades humanas en los ecosistemas de montaña ${ }^{12}$. Bajo este encabezado se organizaron, al menos, cinco reuniones en las que se fue delimitando paulatinamente los alcances de las investigaciones del grupo, los aspectos que debían abordarse y la forma de hacerlo.

En la reunión inicial de trabajo, realizada en Salzburgo (Austria) ${ }^{13}$ se definieron, primero y a grandes rasgos, los problemas de las montañas y, segundo, se identificaron los temas prioritarios que debían de investigarse. A este encuentro le siguieron otros cuatro más, celebrados en los años sucesivos en los que se fueron matizando los temas de interés ${ }^{14}$.

En relación con los problemas de las montañas, aunque en Salzburgo se guardaron de hacer generalizaciones pues cada sistema montañoso tiene sus peculiaridades, se identificaron una serie de rasgos en común. El principal era el grado de aislamiento que las montañas tienen que las hace semejantes, salvaguardando las distancias, a "islas en tierra". Este es el causante de los numerosos endemismos que se pueden hallar a nivel local, pero también de algunos de los problemas sociales que en ellas se viven, como puede ser la pobreza económica en la que viven muchos de sus habitantes, en gran parte derivada de la intensidad a la que se someten sus delgados suelos. Aunque tampoco este rasgo es generalizable. En el Grupo de trabajo eran conscientes de la existencia de grandes, populosas y boyantes ciudades en zonas de montaña, por encima de los 2.000 metros de

\footnotetext{
12 Posteriormente, se amplió el ámbito de atención a las zonas de tundra.

${ }^{13}$ Realizada entre el 29 de enero y el 4 de febrero de 1973. Véase UNESCO (1973).

${ }^{14}$ Así en 1973 se celebró otra en Lillehammer (Noruega) (Véase UNESCO, 1974); en 1974, en la Paz (Bolivia), (véase UNESCO, 1975a); más tarde en ese mismo año en Kathmandú (véase UNESCO, 1975b), y una última reunión celebrada en Lima (véase UNESCO, 1976).
} 
altitud, como es el caso de Bogotá y ciudad de México, y de otras a no tan alta posición como Río de Janeiro (Brasil), o Jakarta (Indonesia) rodeadas también de elevados cerros.

La verticalidad es otro de los rasgos de este medio directamente unido a la dificultad de acceder a él y al aislamiento anteriormente comentado. Esta contribuye a la diversidad biológica al obligar a las plantas a adaptarse a las diferentes condiciones ambientales de cada piso altitudinal. A esta característica se la conoce como "zonación vertical". Paralelamente, al ser humano ésta le ha sido positiva porque ha podido vivir de la producción obtenida en diferentes pisos o bien especializarse en el cultivo de uno único.

Pero la verticalidad también es la causante de otro de los problemas que padecen las poblaciones de montaña. Está asociada con la pendiente, en algunos casos muy pronunciada, y a la inestabilidad de los terrenos. Una mala gestión de los suelos conlleva su erosión y la pérdida de la capacidad productiva. Enseguida vienen a la mente los deslizamientos de terrenos, deslaves, o técnicamente "movimientos de remoción en masa" que afectan a las poblaciones locales y a las inundaciones de los pobladores situados en las partes bajas de las montañas.

En definitiva, la montaña por las características mencionadas ya en los años setenta se la definía como "medio frágil" y la excesiva presión sobre los abundantes recursos con que se acostumbra a asociarlas podía derivarse en degradación irreversible del medio, bien por la existencia de una abundante población que demanda alimento para vivir, bien por la voracidad de empresas mineras, forestales, energéticas, o turísticas que han explotado, y lo siguen haciendo, hasta la saciedad las riquezas con que se asocia a las montañas ${ }^{15}$.

El Working Group núm. 6 distinguía varias zonas de interés:

1) Las de mayor altitud (high altitude), más de 2.500 metros sobre el nivel del mar, en donde, como se ha mencionado, se podían encontrar asentamientos humanos importantes;

2) Ciertos ecosistemas de montaña en los que la presión humana era de consideración y debían investigarse: entre ellos estaban los de las zonas tropicales y subtropicales (situados entre los $30^{\circ}$ de latitud $\mathrm{N}$ y $\mathrm{S}$ ); los de climas templados, principalmente afectados por actividades como el turismo estacional, ya de invierno como de verano; los sistemas de montañas de climas mediterráneos, en los que pese a que su altitud no era muy elevada están sometidos a la acción de usos tradicionales (agricultura y ganadería), a los recurrentes incendios forestales y a la intromisión de segundas residencias con todas sus necesidades de equipamiento y servicios; un cuarto grupo eran las montañas de zonas áridas y semiáridas, habitualmente muy poco pobladas, pero que la tecnología ha permitido extender la urbanización y en los que las empresas, principalmente extractoras, están acabando con sus delicadas características.

3) Finalmente, se consideraban aquellos sistemas montañosos afectados por la tecnología a gran escala (minería a cielo abierto, explotación de recursos energéticos e hídricos, y la construcción de vías de comunicación).

\footnotetext{
${ }^{15}$ Para el caso mexicano actual puede leerse Sunyer y Monterroso (2014), en el que se ofrece un amplio panorama de los problemas que se viven en las montañas mexicanas.
} 
La montaña em los programas internacionales de la UNESCO (1972-2002): del Man and Biosphere...

El punto de vista que aplicó el Working Group núm. 6, como el resto de los participantes en el programa $M A B$, era de carácter naturalístico, con énfasis en lo que se ha denominado "paradigma ecológico" o "ecosistémico", y de corte neomalthusiano, en los que preocupaba la presión humana sobre los recursos y creían necesario evaluar la capacidad de carga de los ecosistemas. Preocupaba también impulsar el desarrollo -económico - como único medio de acabar con la pobreza y el hambre de estos espacios terrestres, siempre respetando los límites ecológicos del medio. En última instancia, se abogaba por la aplicación de programas de ordenamiento territorial (Regional planning) como el único medio para solventar la situación de crisis que se preveía ${ }^{16}$.

Son, como podemos colegir, los mismos puntos de vista que habían orientado la investigación sobre los "límites del crecimiento" encargada por el Club de Roma a los técnicos del Massachusets Institute of Technology (MIT) y el tema central que condujo a la organización de la Conferencia Internacional sobre el Medio humano, de Estocolmo en $1972^{17}$. También son los que llevaron, desde el World-Watch Institute, a Erick Eckholm y su renombrado artículo (The Deterioration of Mountain Environments, 1975) a criminalizar las poblaciones de montaña tachándolas de irresponsables -ecológicamente hablando- ${ }^{18}$. Parecía claro que no se podía seguir creciendo ilimitadamente sobre una base finita.

\section{El dilema himalayo y la geoecología}

Se suele achacar al enfoque geoecológico la alarma que pareció levantarse en los años setenta que culminó en lo que se ha dado en denominar "Teoría de la degradación ambiental de los Himalayas" (Theory of Himalayan Environmental Degradation) o "dilema himalayo" (Himalayan dilemma) ${ }^{19}$. Con este término se hacía referencia al aparentemente inevitable deterioro de los ambientes de montaña sometidos a una creciente presión demográfica y la explotación indiscriminada de sus recursos naturales. Por la mayor atención que despertaba esa gran cadena montañosa (HimalayaHindukush- Karakorum), recibió el nombre anteriormente citado, aunque no era la única. También los Andes formaban parte de esa preocupación ${ }^{20}$.

La geoecología era el punto de vista propuesto por el geógrafo alemán Carl Troll para abordar, desde la geografía, los estudios del medio natural, y particularmente los de la alta montaña. Este

\footnotetext{
${ }^{16}$ El término original en inglés es "regional planning" y lo hallaremos frecuentemente en las últimas reuniones de este Grupo de trabajo.

${ }^{17}$ Del estudio encargado por el Club de Roma se derivó el trabajo encabezado por Dennis y Donella Meadows tan conocido The Limits to Growth: A Report for the Club of Rome's Project on the Predicament of Mankind (1972). Sobre la conferencia de Estocolmo puede consultarse la siguiente URL: $<$ https://sustainabledevelopment.un.org/milestones/humanenvironment>.

${ }^{18}$ Eckholm, 1975.

${ }^{19}$ Ives y Messerli, 1989; Allan, 1992; Ives, 1992, Kreutzmann (1998). Ives y Messerli apelan al origen político, económico y social más que ambiental de la alarma generada. Ambos autores trataban de mostrar que muchos de los estudios que se habían realizado sobre la gran cadena montañosa del Himalaya no tenían el rigor necesario para sustentar políticas y que posiblemente se hicieron sin haber ido al terreno. Tratan los autores de equilibrar el estudio geográfico físico con el humano para ahondar en la imagen estereotipada de la crisis ambiental de las montañas en general.

${ }^{20}$ Una versión de algunas de estas lecturas puede encontrarse en Shafi, Razh (1994, en línea).
} 
nombre fue dado por este geógrafo en $1963^{21}$ para referirse al trabajo previo que ya estaba realizando a partir de fotografías aéreas sobre lo que él mismo había denominado desde 1938 "ecología del paisaje"(Landschaftsökologie) ${ }^{22}$.

Algunos autores remontan este enfoque predominante hasta Ratzel y lo extienden hasta Carl Troll, para denominarlo "modelo Ratzel-Troll" "23. En él se otorga considerable importancia al medio físico y en particular a la "geoecología" como causa explicativa de la mayor parte de los fenómenos que se daban en las montañas, incluidos los hechos humanos. Como bien expresaba Allen, la geoecología era "la rúbrica de los científicos que trabajaban extensamente los procesos físicos en zonas de montaña" 24 .

El punto de vista geoecológico conllevó un uso un tanto amplio del concepto de ecosistema, por ejemplo, para aludir a las zonas de montaña como un único ecosistema cuando, en realidad, en las montañas hay múltiples ecosistemas, pero también el de "zonación" entendida altitudinalmente. Todo podía explicarse a partir de esta fórmula. La zonación vertical de la vegetación y de los climas era uno de los "hechos científicos" innegables, que también parecía aplicarse a los cultivos, la ganadería o la propia habitación.

La Teoría de la degradación ambiental de los Himalayas tuvo su reflejo en los numerosos estudios que se realizaron y en el tipo de políticas de desarrollo que se pretendían aplicar o que, de hecho, se aplicaron y que el mismo Working Group núm. 6 del MAB tampoco se podía deslindar. Como explicó años más tarde Jack Ives (2006), una de las personalidades más implicadas en el estudio del medio de montaña, se vivía en esos años un ambiente de catástrofe en el mundo de la montaña derivado de la Conferencia de Estocolmo y que hacía ver la necesidad de intervenir científicamente en las montañas asiáticas para evitar la crisis humanitaria que, seguro, se iba a vivir en los países próximos a la cordillera de los Himalayas.

Ejemplos de esta preocupación fueron el Manifiesto de Munich, resultado de la reunión organizada en esta ciudad por la agencia alemana para el desarrollo (GTZ) y la UNESCO, en diciembre de 1974, sobre El desarrollo en los ambientes de montaña ${ }^{25}$, y también algunos de los proyectos que se suscitaron en aquellos años como la Misión para la investigación de los ecosistemas del Himalaya, que dio lugar a dos informes, el Informe India y el Informe Nepal, que estuvo financiada por el Ministerio de Cooperación Económica de la antigua República Federal alemana ${ }^{26}$. En ellos

\footnotetext{
${ }^{21}$ Fue en el Simposio sobre "Sociología vegetal y ecología del paisaje” de Stolzenau/ Wesser (Alemania) los días 8 al 11 de abril de 1963, ciudad que había acogido anteriores simposia sobre cuestiones de botánica donde Troll parece usar por primera vez este concepto (Tüxen, 1968, p. 42).

${ }^{22}$ Así lo expresa en un breve párrafo transcrito de la obra mencionada: "Air photo interpretation is to a large degree

"landscape ecology". Only 28 years later, the author proposed the term "Geoökologie" (Geoecology) as a synonymous designation for international use" (Troll [1975], 1988)

${ }^{23}$ Aunque el mismo Troll reconocía como antecedente al propio Humboldt (Troll, en Tüxen, 1968, p. 40)

${ }^{24}$ Allan, 1994, p. 6. Este autor habla del "paradigma geoecológico". Hace mención este autor a un ensayo de Ratzel titulado los límites y los cinturones altitudinales (Höhengrenzen und Höhengürtel), publicado en 1889 en Zeitschrift des Deutschen und Oestereichischen Alpenvereins y que luego expuso en Contribuciones a la Geografía física, etnografía y antropogeografía, un breve texto del geógrafo alemán en el que dentro del estudio de la montaña se hace referencia a esos dos términos, entre otras consideraciones. Véase Ratzel,1908, p. 174.

${ }^{25}$ Sobre el "Manifiesto de Munich" véase Ives, 1981; 2006, p. 228 y ss.

${ }^{26}$ Rieger, 1976a y 1976b. El nombre de la comisión Himalayan Ecosystems Research Mission
} 
se habla de dos de los grandes problemas detectados en sendas grandes regiones de los Himalayas que son la deforestación y la erosión del suelo, preludio de un escenario de Gran desastre ${ }^{27}$.

Los estudios y obras dedicadas al estudio de las montañas aparecidos desde los años setenta hasta bien entrado el decenio de los ochenta participaron de este ambiente. Así, al trabajo antes citado de Eckholm (1975) u otras obras del mismo autor ${ }^{28}$, se añaden trabajos con parecida orientación, como el de J. S. Lall The Himalaya. Aspects of Change (1981), o el documental de Sandra Nichols, The fragile mountain (1982) ${ }^{29}$, que nos devuelve a un entorno presuntamente idílico, en equilibrio delicada e históricamente conseguido, cuya alteración iba a deparar en hambrunas y muertes. O la recién inaugurada revista Mountain Research and Development ${ }^{30}$, de la International Mountain Society $^{31}$. Era el círculo vicioso "crecimiento demográfico-degradación ambiental-pobreza" que parecía caracterizar las zonas de montaña, especialmente las más populosas ${ }^{32}$.

\section{La crisis ambiental y humana en las montañas, la búsqueda de nuevos enfoques}

A pesar de que la criminalización hacia los habitantes de las montañas predominó en la mayoría de las publicaciones de aquellos años y se extendió hasta los años 90, desde el Working Group se fue tratando de matizar esta posición en las sucesivas reuniones de trabajo que se llevaron a cabo. Sus miembros eran conscientes de que era difícil entender la montaña y sus procesos únicamente desde la ecología y que era necesario buscarse aproximaciones de corte integral e interdisciplinario que permitieran adentrarse en la naturaleza compleja de los temas que debían de abordarse en su

\footnotetext{
${ }^{27}$ Podemos añadir la conferencia organizada en Canterbury (Nueva Zelanda) sobre los recursos de montaña, en 1977 de la que se derivó un documento que venía a ser una guía "ecológica" para la gestión de los recursos naturales de la alta montaña. Véase Dasmann y Poore, 1977.

${ }^{28}$ Como puede ser Losing ground. Environmental stress and world food prospect (1976), en la que se apuesta por la necesaria planeación del territorio para evitar la pérdida de suelos y, entre otras cosas, paliar la reducción en la producción de alimentos

${ }^{29}$ Que puede encontrarse todavía en la web. Véase Nichols, 1982.

${ }^{30}$ La revista Mountain Research and Development, de la International Mountain Society ofrece una buena muestra de estas opiniones. Entre ellos, la preocupación por la inestabilidad a que el ser humano estaba sometiendo los recursos del planeta debido a un uso indiscriminado de los mismos, en particular del recurso suelo Particularmente, destaca el discurso que exhibió Jack D. Ives como director de la International Mountain Society, la institución que financia Mountain Research and Development, en el artículo aparecido en su segundo número. Con el título "Crisis in the Mountains", Ives hace un recuento de los problemas que se vivían en las montañas, especialmente las del Himalaya, por el incremento en la población que vive en ellas y que depende de ellas y de sus recursos (Ives, 1981). Otra muestra es uno de los números especiales de la revista citada dedicado a la "estabilidad e inestabilidad de los sistemas de montaña", resultado de un taller celebrado en Berna-Riederalp (Suiza) entre el 14 y el 19 de septiembre de 1981 (Véase International Mountain Society, 1983).

${ }^{31}$ Sobre la International Mountain Society puede verse más adelante en la sección "Fuentes digitales para el estudio de la montaña".

${ }^{32}$ Este punto de vista de corte ecológico se mantendrá durante unos años más. Por ejemplo, la obra citada de Singh, 1992, vuelve a hacer referencia a la excesiva presión que las poblaciones de montaña ejercen sobre los recursos, con lo cual de desenvolvía un círculo vicioso que conducía irremisiblemente a las poblaciones hacia la pobreza y la desnutrición, y a los suelos a ser sobreexplotados agrícolamente y en última instancia perder su fertilidad y ser susceptibles de erosionarse. En particular, uno de sus capítulos, debido a Jack D. Ives (1992) explicaba como la erradicación de la malaria en Nepal había permitido el aumento de la población y con ella la sobreexplotación del suelo agrícola o la extensión de esta actividad sobre zonas forestales o a suelos de mayor pendiente. Ives (2012) lo comentaba así: quizás los científicos ya habían entendido la excesiva atención que se le dio al enfoque geoecológico, pero no las agencias nacionales y regionales de muchas zonas del mundo.
} 
estudio. Así, se opinaba que "es evidente que ni los enfoques sectoriales de investigación, ni las acciones de desarrollo, son adecuadas para proporcionar pautas de gestión y lograr buenos resultados en lo económico, ni para preservar la productividad futura en las zonas de montaña" 33 .

De ahí se puede entender el cambio en el título de los informes de este Grupo de trabajo al introducir el término de "investigación ecológica integrada" ("integrated ecological research..."), con la idea de "predecir la interacción hombre- ambiente". No obstante, llevarlos a cabo conllevaba la necesidad de resolver el espinoso asunto de los recursos "tecnológicos, financieros y humanos" de estas regiones ${ }^{34}$.

La orientación hacia los enfoques ecológicos integrados dio pie a diversas iniciativas, entre ellas las propuestas de establecer un centro de investigación en fisiología y patología de altitud ("highelevation"); de creación de un instituto de ecología de montaña ${ }^{35}$; de configuración de reservas de la biosfera (un nuevo tipo de área de protección impulsada desde la UNESCO en esos años) y de basar los estudios e iniciativas de desarrollo a partir de una nueva unidad de gestión ambiental, las cuencas hidrográficas. Particularmente estas dos últimas: cuencas hidrográficas y reservas de la biosfera permitirían obtener unidades geográficas que sirvieran como línea de base para entender la serie de transformaciones que el ser humano había ocasionado o estaba ocasionando en este peculiar medio.

La investigación ecológica integrada habría de culminar en iniciativas políticas de planeación regional (Regional Planning) para las que los Himalayas y, especialmente, los Andes parecían ser las regiones privilegiadas. Ambas podían ejemplificar la nueva manera de gestionar los espacios de montaña y la posibilidad de integrar los avances de los demás proyectos del MAB ${ }^{36}$. Para el caso andino, permitiría organizar los territorios de los países afectados a partir de sus "cuencas hidrográficas", de manera que todos los ecosistemas comprendidos entre las montañas y el mar, ya fuese en la vertiente atlántica como en la del Pacífico, y desde Colombia a Valdivia (Chile) y Neuquén (Argentina), pudiesen beneficiarse de las investigaciones científicas. Así los proyectos 1, 3, 4 y 5 del MAB (a saber, "bosques tropicales y subtropicales"; "tierras de pastoreo"; "zonas áridas"; "ríos, lagos y estuarios" y "zonas costeras") eran algunos de los que iban a poder integrarse en el proyecto 6 "ecosistemas de montaña".

Como puede colegirse, tras los Grupos de trabajo del $M A B$ había un proyecto ambicioso de desarrollo principalmente económico impulsado principalmente por los "países desarrollados" y que iba a tratar de hacerse factible en algunas regiones de montaña del mundo, como fueron principalmente las de los Andes y los Himalayas.

Tal como se ha tratado de explicar, en realidad, el esfuerzo realizado desde los años setenta en pro de la montaña, más allá de ese momento inicial, no fue en realidad especialmente fructífero, en el sentido de que a pesar de las propuestas y estudios que se generaron poco sirvió realmente para frenar el deterioro de las zonas de montaña ni para comprender la diversidad de las formas de vida

\footnotetext{
33 UNESCO, 1975b, p. 6.

${ }^{34}$ Bhatt, 1976, p. 232.

${ }^{35}$ Algunas de las organizaciones e instituciones como "The Mountain Institute", la "InternationalMountain Society", ICIMOD..., creadas en los años 80 podrían responder a estas iniciativas (véase apartado final "Fuentes para la investigación").

${ }^{36}$ Así se desprende de lo argumentado en Ives, 1977. Véase también Sethi, 1976.
} 
La montaña em los programas internacionales de la UNESCO (1972-2002): del Man and Biosphere...

que albergaban. El cambio de actitud hacia los problemas de las montañas provino al principio de los años ochenta y fueron algunos de los que más se habían implicado los que decidieron reflexionar sobre lo avanzado. El artículo de Ives y Messerli titulado "Stability and unstability of mountain ecosystems", prólogo de una reunión tenida en Berna (Suiza) sobre este tema (1984) ${ }^{37}$, advierte a los investigadores de las montañas que el enfoque hasta aquel momento aplicado no era el más indicado para entender las relaciones de las sociedades con este medio, que era necesario tener mayores datos.

\section{Cambio de rumbo: hacia la Cumbre Mundial de Río 1992}

El decenio de 1980 fue un momento de cambios en la perspectiva de los estudios de la montaña que fueron propiciados por una conjunción de factores. En primer lugar, de los intereses de distintas organizaciones e instituciones. A los estudios y debates habidos en los años setenta, alimentados en gran parte por el programa $M A B$, se añade por ejemplo el cambio de rumbo que desde la Unión Geográfica Internacional (UGI) se empezó a dar hacia los estudios más aplicados:

Si en 1968 a propuesta de Carl Troll se creó en la UGI la Comisión de Geoecología de Alta montaña que animó la realización de numerosos estudios desde este enfoque, la enfermedad primero y el deceso del geógrafo alemán en 1975, condujo a la presidencia de tal Comisión a Jack D. Ives, en 1972, a la sazón coordinador del Grupo de trabajo núm. 6 del MAB, académico de la Universidad de Colorado (EE.UU.) y responsable entonces de la publicación Arctic and Alpine Research. En 1976, con ocasión del Congreso Geográfico Internacional, de Moscú (U.R.S.S.), se celebró el simposio de la Comisión y se decidió cambiar su nombre por el de Geoecología de Montaña, pues empezaba a ser de interés la montaña por debajo del límite del arbolado y de las nieves, donde se encuentran los temas relacionados con el habitar. El tema de tal Comisión se renovó en sucesivos simposios hasta 1988 .

Por su parte, la Universidad de las Naciones Unidas (UNU) se había implicado considerablemente desde el año 1978 con un proyecto titulado Highland-Lowland Interactive Systems como parte de un programa titulado Uso y gestión de los recursos naturales en los trópicos húmedo y subhúmedo (1977) en el que estaba Bruno Messerli ${ }^{38}$. La asociación Ives desde el MAB y la UGI con Messerli dio varios frutos que orientaron una parte de los estudios de montaña.

Con apoyo de la UNU también se fundó la International Mountain Society, organización que pretendía agrupar los investigadores sobre la montaña y vincularla con la necesaria presión política hacia las instituciones internacionales. En su seno se publicó desde 1981 hasta la fecha la revista Mountain Research and Development, una de las publicaciones más representativas de las investigaciones sobre la montaña.

\footnotetext{
${ }^{37}$ Ives y Messerli, 1984. Posteriormente Hewitt (1988), Allan (1992), Kreutzmann, 1998.

${ }^{38}$ Bruno Messerli empezó a colaborar en un proyecto de montaña de la UNU en los Himalaya y Bangladesh titulado "Mountain Ecology, Water Resources, Land Degradation and Natural Hazards", posteriormente titulado "Mountain Ecology and Sustainable development" (Ives, 2012, p. 19).
} 
En este contexto, se favorecieron las transformaciones que iban a darse en estos años también en la organización geográfica. La UGI en 1988 modificó el nombre de la Comisión anterior por el de Geoecología de montaña y de desarrollo de recursos (1988-1992) y a partir de la Cumbre Mundial sobre el Medio Ambiente y el Desarrollo de Río de Janeiro (Brasil), 1992 cambió nuevamente por el de Geoecología de montaña y desarrollo sostenible (1992-2000).

Con motivo de los preparativos de la Cumbre citada, se constituyó un grupo informal denominado Mountain Agenda ${ }^{39}$ que tenía como objetivo presionar políticamente para que entre los temas prioritarios de discusión se incluyera el de las montañas. Paralelamente, desde la UNU se estaba trabajando en un informe que sirviera para apoyar esta iniciativa. De ahí resultó la obra firmada por Peter B. Stone The State of the World's Mountains: a Global Report $(1992)^{40}$, cuya versión preliminar fue distribuida entre los delegados internacionales que iban a participar en Río. En aquel momento era el único informe relativo a la situación de este espacio en el mundo ${ }^{41}$. Este documento, junto con Appeal for the Mountains, permitió situar a la montaña en una situación equivalente a otros de los principales problemas del mundo, como la conservación de la biodiversidad, y el cambio global ${ }^{42}$.

\section{La montaña en el capítulo 13 de la Agenda 21}

La Agenda 21 fue aprobada en la Cumbre de Río'92 y con ella el capítulo 13 que lleva por título "Gestión de los ecosistemas frágiles: desarrollo sostenible de las zonas de montaña". En ella se describen a grandes rasgos los problemas que afectan a las montañas y a sus habitantes. Sin embargo, sólo dos aspectos son los que se consideran relevantes: 1) la generación y reforzamiento del conocimiento ecológico y la sostenibilidad de los ecosistemas de montaña; 2) la promoción del desarrollo integrado de las cuencas hidrográficas y de formas alternativas de medios de vida. Nuevamente, la visión objetual y utilitaria de la montaña traslucía en ambos proyectos. En el primer punto básicamente interesaban los recursos que este medio poseía, y en el segundo volvía a emerger el concepto de "cuenca hidrográfica", presente en los reportes del grupo de trabajo 6 del MAB, y una cierta penalización de las formas de vida que desde hace quizás milenios han caracterizado el habitar de las alturas. Lo positivo es que se empezaba a visualizar a las montañas y sus problemas al lado de los otros grandes problemas que afectan a la humanidad.

El cambio se irá reflejando progresivamente tanto en las publicaciones que se irán sucediendo como dentro de los organismos internacionales. Un ejemplo son los informes de la Comisión para el Desarrollo Sostenible del Centro Económico y Social de las Naciones Unidas de 1995 y 1997, si los comparamos con los objetivos del propio capítulo 13 de la Agenda $21^{43}$. Si en este capítulo se habla de la necesidad de conocer los recursos de las montañas e implementar programas de control

\footnotetext{
${ }^{39}$ Formada por académicos, por responsables de la FAO, de Organizaciones no gubernamentales. Véase al respecto Price et al., 2004.

${ }^{40}$ La Comisión para el Desarrollo Sostenible de las Naciones Unidas tuvo como encargo hacer el seguimiento de los temas desarrollados en la Agenda 21, entre ellos el de la montaña. La información sobre la actividad realizada por la UNU se explica en uno de estos documentos (CSD, 1995, Párrafo 84 y ss.). Sobre el informe véase Stone (1994)

${ }^{41} \mathrm{Si}$ bien su alcance geográfico tampoco fue tan extenso. Se abordaron principalmente las regiones andina, Apalaches y las montañas de la antigua URSS y algunos sistemas montañosos más (las montañas de Hengduan-Xi, los Pirineos, Sierra Nevada -California-para mostrar las raíces históricas de la pobreza de las zonas de montaña (Miller, 1994)

${ }^{42}$ CSD (1995, párrafo 1984, 84 y ss); Price (1998, s/p. y 2004).

${ }^{43}$ Véase más abajo en el apartado "Conferencia Río+5".
} 
de cuencas hidrológicas a partir de la forestación de las cabeceras de cuenca, en 1995 se abre la puerta a abordar la gestión integral de los recursos naturales, los problemas de la interacción entre las poblaciones rurales y los recursos en términos de vida sostenible. Finalmente, en 1997, se da pie a muchos de los temas que deben de abordarse en una Agenda de Montaña para el nuevo milenio. Se dice explícitamente que han aparecido nuevos temas en los cinco años transcurridos desde la Cumbre de Río y que, en esos momentos, 1997, se debía de poner énfasis "en la conservación, en la cultura, en los valores sagrados y en la diversidad paisajística de las montañas". Se habían de superar los enfoques que veían las montañas como meros objetos y entender los valores espirituales que atesoran y que tan importante era proteger la biodiversidad como a las propias culturas que en ella habitan.

Quizás lo más relevante es que muchas de las obras que aparecieron en esos años y en los subsiguientes a la Cumbre de Río pueden ser caracterizadas como geográficas, ya sea por su perspectiva del medio físico, ya del humano. En este sentido, parecían recuperarse al menos parte de la tradición geográfica de los estudios sobre la montaña que ya había desarrollado la geografía regional y humana francesa desde el segundo decenio del siglo XX y que el geógrafo norteamericano Roderick Peattie trasladó a la geografía estadounidense con la obra Mountain geography, a critique and field study (1936).

\section{Lecturas geográficas de la montaña y sus problemas}

En los años previos a la Cumbre de Río se publicaron algunos libros que reflejan todavía el tránsito de los temas desde la geografía física a la humana. Algunos son referentes de los estudios de la montaña como son el de Larry W. Price Mountain and Man. A Study of Process and Environment (1981) que, aunque abordaba los temas de la geografía humana atendía sobre todo a la alta montaña y daba prioridad al enfoque geoecológico, a los aspectos de la geografía física de las montañas. La obra de Price ha sido considerada como heredera del libro de Peattie, considerado como el primer texto en inglés sobre la montaña publicado 45 años antes, que el propio autor consideraba como un trabajo de geografía humana.

Junto al texto de Price otras obras también de orientación geográfica trataban de denunciar las modificaciones del medio de montaña que la modernidad, la afluencia de turismo, las decisiones políticas de los gobernantes y la acción humana a distancia, entre otras, ocasionaban en la biodiversidad y la cultura. Así, la obra de J. S. Lall Himalaya. Aspects of change (1981) se centra principalmente en el papel del ser humano como agente del cambio; cambios que se habían experimentado ya en esos años setenta del medio físico y que afectaba a las poblaciones humanas y sus posibilidades de desarrollarse. Era necesario, creía Lall, armonizar las necesidades humanas con la conservación de este medio tan vulnerable como es el de las altas montañas.

Otros autores alertaban de la pérdida cultural que los procesos de modernización generaban. Así la obra editada por Michael Ch. Tobias Mountain People (1986) ${ }^{44}$ daba fuerza a los aspectos

\footnotetext{
${ }^{44}$ Estuvo precedida de The Mountain Spirit (1979) de Tobias y Drasdo en la que se hace una compilación de textos de autores diversos que han visto en la montaña algo más que el recurso natural necesario para el funcionamiento de nuestras sociedades.
} 
culturales e incluso espirituales de la geografía humana de las áreas de montaña; un mundo en trance de desaparición, tan frágil como la riqueza natural que alberga.

En esos mismos años se ensayaba otra forma de aproximación a la investigación de la montaña alejada del dilema himalayo que Ives y Messerli estaban denunciando desde principios de los años ochenta y se veía necesario acercarse a la realidad humana, social, económica y cultural de los pueblos de montaña ${ }^{45}$. Un libro de gran interés en esta línea es la obra colectiva editada por Allan, Knapp y Stadel, Human Impact on Mountains (1988). Es un texto crítico con la forma como se había abordado con anterioridad este medio ${ }^{46}$ y propone un acercamiento al ser humano con los problemas clásicos como la verticalidad, el cambio de los usos del suelo, la necesidad de cartografía detallada de las zonas de montaña, pero también muchos de los problemas tan actuales como son el turismo, los procesos de modernización agrícola y ganadera, entre otros.

La nueva manera de ver las montañas convivía, necesariamente a finales del decenio, con trabajos como el de John Gerrard Mountain Environments: an Examination of the Physical Geography of Mountains (1990) que, con un enfoque próximo al de Price, ofrecía una visión aplicada de la geografía. El autor era consciente de que para poder paliar muchos de los problemas físicos del medio de montaña, era necesario conocer sus características y procesos, no en vano, en ella se asienta un 10 por ciento de la población mundial, y es un medio vulnerable a las actividades humanas desarrolladas tanto en las montañas como fuera de ellas.

El informe de Stone The State of the World's Mountains (1994), presentado en la Cumbre de Río'92, fue un parteaguas en la forma de entender los estudios de montaña y entre este año y Río+5 (1997) y dio lugar a la parición de nuevos trabajos con una visión menos maniquea del papel de los pueblos de montaña. Así, por ejemplo, en esta dirección salió el libro de Kapoor y Singh Man and Development in Himalayas (1996) quienes apostaban por impulsar proyectos de base local, que tuviesen que ver con las propias necesidades de sus habitantes, y no realizados o forzados por las administraciones públicas de los respectivos países y requeridos por agentes externos; y la obra de Messerli e Ives (1997) Mountains of the World: a global priority ${ }^{47}$. Los trabajos recogidos habrían de servir para la sesión especial de la Asamblea General de la ONU en Nueva York, en 1997, en la revisión quinquenal de los acuerdos de Río’92.

Poco tiempo después, en otra sesión del organismo internacional se acordó dedicar un año internacional, el 2002, a las montañas. Por primera vez un espacio geográfico recibía tal honor ${ }^{48}$. Destaca en esta etapa también el trabajo de Don Funell y Romola Parish Mountain Environments and Communities $(2001)^{49}$, una perspectiva geográfico-humana al estudio de este medio.

\footnotetext{
${ }^{45}$ Ives y Messerli (1989).

46 Por ejemplo, el capítulo de Hewitt, 1988.

${ }^{47}$ Esta obra reunía diferentes ensayos que fueron presentados primeramente en el sitio electrónico de Mountain forum y luego publicados en formato de libro. Posteriormente, fue reeditada y actualizada en 2004 al abrigo del Año Internacional de las Montañas por Martin F. Price et al., 2004. Sobre Mountain Forum, véase Byers, 1998.

${ }^{48}$ Asamblea General de las Naciones Unidas de 1998 en la que se hace la declaración (A/RES/53/24 de 19 de noviembre de $1998<$ https://undocs.org/en/A/RES/53/24>.

49 Se puede obtener a través de plataformas como Scopus, en línea en la siguiente dirección: <https://bidi.uam.mx:6990/10.4324/9780203992067>.
} 
En este recorrido no exhaustivo de textos geográficos recientes sobre la montaña, tres obras finales que merecen destacarse son las siguientes: Environmental change in mountain and uplands (2000), de Martin Beniston, profesor de Geografía en la Universidad de Fribourg (Suiza), reeditada recientemente en 2016. Especializado en temas de cambio global, previamente había publicado Mountain Environment in Changing Climates (1994), de gran interés en momentos como el actual que parecen cumplirse las peores premoniciones con respecto al calentamiento global. La segunda obra es de Jack D. Ives Himalayan perceptions (2004) cuyo subtítulo es indicativo de los nuevos tiempos en el estudio de las montañas: Environmental Change and the Well-Being of Mountain people. Finalmente, la obra de Price, Byers y otros Mountain Geography: Physical and Human Dimensions (2013) una revisión muy necesaria y actualizada de la obra ya mencionada de Larry W. Price (1981) que marca el curso de las investigaciones en el marco de esta especialidad.

\section{Por una ciencia de las montañas: ¿Geografía de la montaña o montología?}

Todas las obras referidas anuncian la emergencia o reemergencia de una especialidad que ha recibido al menos dos nombres y ambos con visos de persistir. Uno primero "geografía de la montaña", un término empleado puntualmente por el geógrafo francés Raoul Blanchard y que puso en boga el norteamericano Roderick Peattie, como se ha señalado ${ }^{50}$. El segundo es "montología", que fue utilizado por Frank Davidson en Munich en 1974 y posteriormente figuró en un artículo de S. Neustadtl titulado "Montology: Ecology of mountains" $(1977)^{51}$. Si bien las obras señaladas anteriormente pueden incluirse dentro del campo de la "geografía de la montaña" sólo la de Price et al. 2013 ha vuelto a emplear como tal este término, aunque anteriormente, desde 1982 hasta tiempos recientes, diversos artículos publicados en prestigiadas revistas geográficas lo rescatan y, en cierta manera defienden como campo disciplinario ${ }^{52}$.

Por su parte, con respecto al término "montología", algunos especialistas en el estudio de la montaña defendieron la necesidad de una "ciencia de la montaña", como la que existía para los océanos y otros medios. Uno de sus impulsores fue Dereck Denniston en una obra de divulgación del World Watch Institute, High Priorities: Conserving Mountain Ecosystems and Cultures ${ }^{53}$ quien clamaba por una disciplina que aunara todos los estudios sobre este medio independientemente de su ámbito disciplinario. Han sido investigadores como Jack Ives, Robert E. Rhoades, y algunos de

\footnotetext{
${ }^{50}$ Sobre el término "géographie de la montagne", Blanchard lo usó en diversas reseñas publicadas en la Revue de Géographie Alpine, órgano de comunicación del Institut de Géographie Alpine que fundó en 1913, por ejemplo, Blanchard, 1927a, p. 166, y 1927b, p. 370.

${ }^{51}$ Neustadtl, 1977. Para la referencia de Davison véase Sarmiento (En publicación).

${ }^{52}$ Stadel, 1982; Soffer, 1982; Meyzenq, 1996; Friend, 1999; Smethurst, 2000; Funnell y Price, 2003; Bishop, 2009; y por supuesto la obra de Price, Byers et al., 2013. Aparte de algunos números especiales de revistas geográficas dedicadas a la montaña, hay que señalar que la Asociación Americana de Geógrafos, a propuesta de D. Friend, creó un grupo de investigación en geografía de la montaña. El ejemplar de 2017 (vol. 107, núm. 2) de sus Annals está íntegramente dedicado a ellas.

${ }^{53}$ Denniston, 1996, p. 12.
} 
los que conformaron la International Mountain Society quienes propusieron con el término "montología" conformar una disciplina propia para el estudio de las montañas ${ }^{54}$.

En cualquier caso, con el nombre que se acabe definiendo, la comprensión de la montaña en toda su diversidad física, biológica y humana requiere de puntos de vista integradores que contribuyan a paliar los problemas que puedan tener tanto sus habitantes como los que viven en lugares próximos y que además apoyen su preservación. Y, sobre todo, es más necesario que nunca que se atienda a aquellos sistemas montañosos como los que abundan en muchos lugares del mundo, como gran parte de Europa, México o toda Centroamérica, que sin tener la vistosidad, y sublimidad de las grandes cadenas de montañas conforman el día a día de cerca de ese 10 por ciento de la población mundial que viven en ellas, a la que no le llegan las políticas gubernamentales de apoyo y sí los inconvenientes de los megaproyectos mineros, energéticos o de infraestructuras.

La geografía ha cambiado sustancialmente en los últimos veinte o treinta años y ha ampliado su campo disciplinario a ámbitos en los que los límites con otras áreas de las ciencias sociales se vuelven cada vez más difusos. Así ha pasado en la geografía cultural, en la histórica y en la ambiental, por mencionar tres de ellos. Por lo que un campo llamado geografía de la montaña podría tener un alentador futuro.

Vale la pena finalizar con una reflexión de uno de los pioneros de los estudios geográficos de la montaña, Raoul Blanchard, quien calificaba este campo -el de la geografía de las montañas-como "una de las disciplinas más difíciles y apasionantes que hay en la geografía" 55 .

\section{Fuentes digitales para el estudio de la montaña}

Hasta aquí, independientemente de las referencias citadas a lo largo del texto, hemos encontrado varios organismos, instituciones e investigadores que se han dedicado o han promovido los estudios sobre la montaña. Muchos se derivan del primer programa de la UNESCO Hombre y Biosfera $(M A B)$, pero otros han ido surgiendo a medida que iban aumentando los temas de interés de las montañas. Sin ánimo de querer abarcar todas las fuentes disponibles, queremos señalar las que pueden ser más útiles al investigador.

\section{Organización de las Naciones Unidas (ONU)}

De este organismo internacional se pueden consultar varias páginas de las diferentes cumbres mundiales que se han celebrado vinculadas con el medio ambiente o relacionadas con la actividad promovida por sus agencias, como la UNESCO y la FAO, principalmente. Estas son algunas de ellas:

ONU/ UNESCO. Programa Hombre y Biosfera (Man and Biosphere, MAB)

\footnotetext{
${ }^{54}$ Así lo explica Sarmiento (2010, p. 188) y Sarmiento (En publicación). Como indica este mismo autor este término figura en el Oxford English Dictionary. También este autor es de los que ha promovido este término con asiduidad (Sarmiento, 2000).

${ }^{55}$ Blanchard, 1927b, p. 370.
} 
La montaña em los programas internacionales de la UNESCO (1972-2002): del Man and Biosphere...

En el portal de la UNESCO, desde la Biblioteca digital ${ }^{56}$ podemos acceder y descargar la mayoría de los informes (Report) que se han publicado en relación con el programa Hombre y Biosfera, la mayoría en dos de los idiomas entonces oficiales, en inglés y francés y cuyas referencias se encuentran en la bibliografía de este texto.

Cumbre Mundial sobre el Medio Ambiente y el Desarrollo, Río de Janeiro de 1992

Específicamente, conviene mencionar el capítulo 13 de la Agenda 21, también conocido como Agenda de Montaña 1992, un documento en el que, como se ha dicho, por primera vez se incluía a este medio. Este capítulo fue producto de la Mountain Agenda, un grupo informal que reunía a distintos especialistas y responsables de organismos involucrados en el estudio de las montañas (UNU, IMS, IUCN, UGI entre otros) desde un enfoque amplio, como el que se estaba orientando desde la UGI, es decir, no solamente la alta montaña sino aquella en la que habitaba el ser humano $^{57}$. La Mountain Agenda era, finalmente, un grupo de presión que no solamente medró para que se aprobara el citado capítulo, sino que también se involucró en su aplicación posterior. La aprobación de la Agenda 21 abrió la posibilidad de desarrollar políticas regionales, interregionales y mundiales de montaña ${ }^{58}$.

\section{Cumbre de la Tierra Río+5}

La revisión de los acuerdos alcanzados en Río tuvo una primera evaluación cinco años más tarde en una sesión especial de la Asamblea de las Naciones Unidas en Nueva York más conocida como Río+5. Había que mantener la llama prendida en 1992 y desde diferentes instituciones oficiales y no oficiales se trabajó en este sentido. La obra clave en estos años fue Mountains of the World: $a$ Global Priority, de Jack D. Ives y Bruno Messerli (1997).

Entre 1992 y 1997, el Comité para el Desarrollo Sostenible (CSD, por sus siglas en inglés), instancia del Consejo Económico y Social de la ONU para hacer el seguimiento de la aplicación de la Agenda 21, publicó algunas disposiciones de cara a hacer efectivos los proyectos de sostenibilidad en las áreas de montaña. Dos de los informes más interesantes fueron Review of Sectorial Clusters, Second Phase: Land, Desertification, Forests and Biodiversity "Managing Fragile Ecosystems: Sustainable Mountain Development" en el que se describe los avances en la aplicación de la Agenda $21^{59}$ (10 de febrero, E/CN.17/1995/5); y el segundo apareció en 22 de enero de 1997 con el título Overall Progress Achieved since the United Nations Conferencie on Environment and Development (E/CN.17/1997/2/Add.12), y el mismo subtítulo (Managing fragile Ecosystems... ${ }^{60}$. Es en este segundo Informe donde se ve reflejada la influencia del grupo Mountain Agenda. No obstante, en la resolución final de la Asamblea General Programme for further implementation of Agenda 21 (A/RES/S-19/2 de 19 de septiembre de 1997) las referencias

\footnotetext{
$56<$ https://unesdoc.unesco.org>.

${ }^{57}$ Ives y Messerli, 2009, p. 353; Ives, 2012.

${ }^{58}$ La Agenda 21 puede consultarse en <https://sustainabledevelopment.un.org/content/documents/Agenda21.pdf .

${ }^{59}$ CSD, 1995 <https://www.un.org/ga/search/view_doc.asp?symbol=E/CN.17/1995/5\%20\&Lang=E >..

${ }^{60}$ CSD, $2000<$ https://www.un.org/ga/search/view_doc.asp?symbol=E/CN.17/1997/2/Add.12\%20\&Lang=E >
} 
a la montaña son pasajeras y vinculadas a otros aspectos de interés, por ejemplo, la preservación del agua dulce, la lucha contra la degradación de suelos etc. ${ }^{61}$.

Cumbre Mundial sobre el Desarrollo Sostenible, Johannesburgo (26 de agosto al 4 de septiembre de 2002)

El informe general de la Cumbre Mundial sobre el desarrollo Sostenible de Johannesburg 2002 (26 de agosto a 4 de septiembre) (A/CONF.199/20*) puede descargarse desde la biblioteca digital de las Naciones Unidas ${ }^{62}$. En él se recogen las resoluciones adoptadas en la "Declaración política de Johannesburgo" y un "Plan de aplicación de las decisiones adoptadas en la Cumbre". Es en esta segunda parte donde se hallan algunas referencias a las montañas, principalmente en el apartado IV titulado "Protección y gestión de la base de recursos naturales del desarrollo económico y social". En él se alude a la necesaria protección de los ecosistemas, entre ellos los de montaña, la gestión integrada de suelos, agua y recursos vivos. En el numeral 42 se destaca su función en la preservación de la biodiversidad, el ser fuente de cuencas hidrográficas y se habla de su vulnerabilidad ante los efectos del calentamiento global.

En relación con sus habitantes, en el documento que estamos revisando se menciona la necesidad de promover "programas, políticas y enfoques" que de forma integral ayuden a erradicar la pobreza; que ayuden frente a la deforestación, la degradación de suelos, la alteración del régimen fluvial; que contribuyan a reducir la inequidad social y económica de las poblaciones de montaña frente a las de otros ambientes menos rigurosos; que promuevan proyectos a escala teniendo en cuenta las especificidades del ámbito es que se encuentran. Se trata de hacer partícipes las comunidades que en ellas viven en el proceso de toma de decisiones sobre proyectos que las afecten. Finalmente, se busca implementar mecanismos financieros para el desarrollo de proyectos que, a la vez que enfrenten a la pobreza, contribuyan a una mejora de la calidad de vida. Hace una referencia explícita al espíritu de los proyectos promovidos con motivo del 2002 Año Internacional de las Montañas.

Conferencia de las Naciones Unidas sobre Desarrollo sostenible, Río de janeiro (Río + 20)

A veinte años de la emblemática Cumbre Mundial de Río'92, se hicieron diversas revisiones de los acuerdos plasmados en la Agenda 21, a los cinco, diez y veinte años de su aprobación. La Conferencia Río+20 se celebró en Río de Janeiro (Brasil) entre el 20 y el 22 de junio de 2012 y los resultados se pueden hallar en el Informe de la Conferencia de las Naciones Unidas sobre el Desarrollo Sostenible (World Summit on Sustainable Development, WSSD) ${ }^{63}$.

En este documento se habla de las montañas en un inciso específico así denominado (numerales 210 a 212), dentro del apartado V "Marco para la acción y el seguimiento", subapartado A "Esferas temáticas y cuestiones intersectoriales". No añade mayor información a la que ya se ha comentado en las otras conferencias y cumbres y, como siempre, se alienta "a los Estados a que adopten una

\footnotetext{
$61<$ http://undocs.org/A/RES/S-19/2>.

$62<$ https://digitallibrary.un.org/record/478154>

${ }^{63}$ ONU, $2012<$ https://sustainabledevelopment.un.org/index.php?page=view\&type $=111 \& n r=1358 \& \mathrm{menu}=35>$.
} 
La montaña em los programas internacionales de la UNESCO (1972-2002): del Man and Biosphere...

visión a largo plazo y enfoques holísticos" para encaminar a las poblaciones de montaña hacia el desarrollo sostenible.

\section{2, Año Internacional de las Montañas ${ }^{64}$}

La declaración de 2002 Año Internacional de las Montañas se entendía como una buena oportunidad para inducir la generación de proyectos que, efectivamente, condujeran al desarrollo sostenible en las zonas de montaña. Así se explicaba en el documento de la Secretaría General de la Comisión para el Desarrollo Sostenible en su octava sesión ${ }^{65}$. También hallamos algunos portales y una publicación que ofrecen una visión de los objetivos y alcances de esta celebración mundial. Por ejemplo, en uno de ellos se reflexiona sobre la importancia de los años internacionales y se aconseja dedicar 2002 como tala las montañas ${ }^{66}$. La declaración se halla en la resolución A/RES/53/24, de 19 de noviembre de $1998^{67}$.

El año internacional fue promovido desde la FAO en colaboración con el Programa ambiental de las Naciones Unidas (PNUMA), el Programa de las Naciones Unidas para el desarrollo (PNUD) y la UNESCO, entre otras agencias. El informe que se elaboró como contribución a este acontecimiento lleva por nombre Mountain Watch. Environmental Change and Sustainable Development in Mountains, editado por Simon Blyth $(2002)^{68}$.

De las actividades implementadas a lo largo de ese año, destacan la Cumbre Internacional sobre la montaña (Bischkek, Kirghizistan) entre el 28 de octubre y el 1 de noviembre de $2002^{69}$. El reporte de lo debatido en la Cumbre puede encontrarse en el documento de la Asamblea general de las Naciones Unidas (A/58/134) de 11 de Julio de $2003^{70}$. Y la información sobre el evento puede hallarse en la Plataforma de las Montañas de Bischkek ${ }^{71}$.

También el Programa Hombre y Biosfera de la UNESCO $(M A B)$ realizó varias contribuciones a la celebración de este año internacional ${ }^{72}$. Así, por ejemplo, a partir de un convenio entre $M A B$ y la organización Iniciativa de Investigaciones sobre la Montaña (Mountain Research Initiative, MRI, Berna, Suiza) se impulsó un proyecto comparativo entre diversas áreas naturales de montaña del

\footnotetext{
$64<$ http://www.fao.org/iym/en/>.

${ }^{65}$ CSD, 2000, p. 50-52 <https://sustainabledevelopment.un.org/index.php?menu=1174>. Un buen relato del proceso que condujo desde la Cumbre Rio de Janeiro de 1992 hasta el Año Internacional de las Montañas puede leerse en Price, et al. 2004, p. 5-12.

${ }^{66}$ Se puede ver en Resolutions and Decisions of the Economic and Social Council (E/1998/98). CES, Consejo

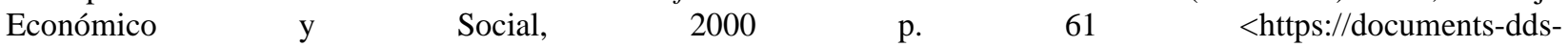
ny.un.org/doc/UNDOC/GEN/N00/472/02/IMG/N0047202.pdf?OpenElement>.

${ }^{67}$ Lo podemos ver en la siguiente dirección: <https://undocs.org/en/A/RES/53/24>.

68 Blyth, $2002<$ https://www.unep-wcmc.org/resources-and-data/mountain-watch--environmental-changesustainable-development-in-mountains>.

${ }^{69}$ Bischkek Global Mountain Summit (BGMS, por sus siglas en inglés). Al respecto, puede verse Price, 2004, p. 9 y ss.

70 <http://www.fao.org/fileadmin/user_upload/mountain_partnership/docs/Report_2003_International\%20Year\%20of $\%$ 20Mountains_2002.pdf $>$.

$71<$ http://www.fao.org/fileadmin/user_upload/mountain_partnership/docs/BM_Platform.pdf $>$.

$72<$ https://unesdoc.unesco.org/ark:/48223/pf0000129467_fre?posInSet=11\&queryId=ba7f91bc-dc8a-4ab7-bd2d$1 \mathrm{~b} 3 \mathrm{c} 24 \mathrm{~b} 6715 \mathrm{f}>$.
} 
mundo. MRI es una de las organizaciones importantes en el estudio actual de la montaña, empresa acogió el Programa Internacional Geosfera-Biosfera (IGBP), el Programa Internacional de la Dimensión Humana del Cambio Global (IHDP) y el Sistema Internacional de Observación terrestre $(\mathrm{GTOS})^{73}$.

El proyecto Alianza de las montañas (Mountain Partnership) ${ }^{74}$ es otro producto de la aportación $\operatorname{del} M A B$ a la celebración. Alianza de las montañas es una organización fundada en 2002 que agrupa a representantes de diversos países, asociaciones intergubernamentales y otras, implicadas en proyectos de desarrollo y de conservación en regiones de montaña de todo el mundo. Actualmente incluye a más de 390 entidades. Sobre la Alianza se publicó en el Año Internacional de las Montañas el Bali Concept Document in "International Partnership for Sustainable Development in Mountain Regions "75 que se acoge a la recomendación realizada en 1992, en la Cumbre Mundial de Río, en la que el entonces Secretario general abogaba por reforzar la cooperación internacional. En el documento citado se ofrece un amplio listado de posibles miembros participantes a los que habría que añadir los representantes de organismos de gobiernos locales, regionales o nacionales que pudieran servir para formalizar tales redes. Se propone, asimismo, que las experiencias resultantes de esta cooperación puedan ser seguidas por el Comité para el Desarrollo Sostenible de la ONU (CSD, por sus siglas en inglés). Sobre la propia organización, puede hallarse más información en el documento Mountain Partnership Organization, Membership and Governance (2004) ${ }^{76}$ y un panorama general de los documentos básicos se puede hallar en <http://www.fao.org/mountain-partnership/about/basic-documents/en/>. El mismo portal de Mountain Partnership nos remite a otros de las Naciones Unidas ${ }^{77}$.

Finalmente, como último producto de este Año Internacional de las Montañas, se elaboró en colaboración con World Heritage Centre y la International Union for the Conservation of Nature (IUCN) un listado de zonas de montaña, áreas naturales protegidas o de interés natural, que podrían ser nominadas también como patrimonio de la humanidad ${ }^{78}$.

\section{Día Internacional de las Montañas (11 de Diciembre $)^{79}$}

Con respecto a este acontecimiento existen varias páginas disponibles. La que reseñamos es la generada para el año 2019 y remite a diferentes portales de interés, todos ellos relativos a las montañas desde las Naciones Unidas. Por ejemplo, la página de la FAO para ese mismo día <http://www.fao.org/international-mountain-day/es/> y la Plataforma de conocimiento de las montañas ${ }^{80}$ que remite a los retos de la Agenda 2030 para el Desarrollo sostenible. En la Plataforma se hace referencia al párrafo 33 en el que se habla de la relevancia de la gestión de los sistemas naturales (entre los que están las montañas) y su impacto en el desarrollo económico y social. Menciona particularmente el logro 15 (Sustainable Development Goal-SDG15-) en el

\footnotetext{
$73<$ https://www.mountainresearchinitiative.org >.

$74<$ http://www.fao.org/mountain-partnership/en/>.

$75<$ http://www.fao.org/fileadmin/user_upload/mountain_partnership/docs/bali_concept_document.pdf>.

$76<\mathrm{http}$ ///www.fao.org/fileadmin/user_upload/mountain_partnership/docs/1_governance_e_final.pdf >.

$77<$ http://www.fao.org/mountain-partnership/publications/un-documents/en/>.

78 Thorsell y Hamilton, 2002.

$79<$ https://www.un.org/es/observances/mountain-day>.

$80<\mathrm{https}$ ///sustainabledevelopment.un.org/topics/mountains $>$.
} 
La montaña em los programas internacionales de la UNESCO (1972-2002): del Man and Biosphere...

que se reconoce que para 2020 muchos de los objetivos propuestos en Río+20 ya son inalcanzables por el grado elevado de deterioro de muchos sistemas naturales. Sobre las montañas dice:

Unos ecosistemas de montaña saludables son fundamentales para asegurar la provisión de servicios ecosistémicos tanto para las poblaciones de montaña como a las de los valles y llanuras inmediatas. Para el año 2017, el 76 por ciento de estas montañas estaba cubierto por algún tipo de vegetación: 41 por ciento de bosque; 29 por ciento de pastos; y un 6 por ciento de cultivos ${ }^{81}$.

Ahora bien, la degradación de los suelos por desertificación, urbanización, expansión agrícola y ganadera está impactando fuertemente en ellas. Lo más preocupante es la extinción de especies asociada $^{82}$.

Este mismo portal encamina hacia el capítulo 13 de la Agenda 21; hacia la Tercera sesión de la Comisión para el desarrollo Sostenible, en particular la que trata de la gestión de ecosistemas frágiles, pero también a otros temas ${ }^{83}$, como la página de "2002 Año Internacional de las montañas". Una penúltima referencia es al documento "the Future We Want" de la resolución de la Asamblea General de 24 de diciembre de 2009 (A/RES/66/288) que dedica un apartado a las montañas, a su importancia y la necesidad de conservar sus ecosistemas (Párrafos 210 a 212) ${ }^{84}$. Por último, se encuentra un vínculo al documento Transformando nuestro mundo (Transforming our World). La Agenda 2030 para el Desarrollo sostenible, correspondiente a la Resolución $\mathrm{A} / \mathrm{RES} / 70 / 1$.

\section{UGI (Unión Geográfica Internacional)}

La Unión Geográfica Internacional (o IGU por sus siglas en inglés) también ofrece algunas vías para acceder a documentos de las distintas comisiones creadas para el estudio de las montañas.

En primer lugar, los documentos más antiguos sólo se encuentran en formato de papel en distintas bibliotecas, por ejemplo, los diversos Proceedings de los simposios organizados entre 1968 y el año 2000. Sólo a partir de reseñas de los mismos publicadas en revistas de Geografía (Erdkunde, GeoJournal) o, desde 1981 en la revista Mountain Research and Development podemos llegar a conocer su contenido y los temas que se discutieron.

Del año 2000 en adelante, con la creación del portal de la organización ${ }^{85}$, se puede acceder a la actividad de las diversas Comisiones que sobre la montaña existen en esta organización. Pero para documentos algo más históricos es preferible encaminarse hacia Home of Geography ${ }^{86}$, web

\footnotetext{
${ }^{81}$ ONU. Informe de la Secretaría General (Mayo, 2019). Los objetivos a alcanzar se pueden encontrar en ONU. Sustainable Development Goals. Targets \& Indicators, $2016<\mathrm{https} / / / \mathrm{sustainabledevelopment.un.org/sdg15>.} \mathrm{En}$ muchos de los puntos de este indicador 15 se mencionan a las montañas.

82 ONU. Informe de la Secretaría General (Mayo, 2019). Los objetivos a alcanzar se pueden encontrar en ONU. Sustainable Development Goals. Targets \& Indicators, $2016<$ https://sustainabledevelopment.un.org/sdg15>. En muchos de los puntos de este indicador 15 se mencionan a las montañas.

$83<$ https://sustainabledevelopment.un.org/index.php?menu=1421>.

$84<$ https://sustainabledevelopment.un.org/rio20/futurewewant>.

$85<$ https://igu-online.org>

$86<$ http://www.homeofgeography.org>
} 
editada por Giuliano Bellezza, que acopia desde 2000 en su biblioteca física y virtual muchos de los materiales de UGI. Para el caso de la Comisión Diversidad en los sistemas montañosos (Diversity in Mountain systems. Grupo C04.08) encontramos información en los archivos de la comisión. Para llegar a ellos tenemos que entrar en el menú "IGUBodies_e-ARCHIVES", y acceder a los documentos previos al Congreso de Túnez (2008), a los de los años 2008- 2012 (Congreso de Colonia), y finalmente los del período 2012-2016 (Congreso de Pekín).

\section{Otras instituciones}

\section{The Mountain Institute 87}

Inicialmente denominada The Woodlands Mountain Institute fue fundada en 1972 para promover proyectos de colaboración con poblaciones de las dos cordilleras más amplias y elevadas del mundo, los Andes y los Himalaya. En su página los responsables de la organización se jactan de ser la única sin ánimo de lucro que trabaja en la conversión de economías locales hacia la sustentabilidad a la vez que se protege su cultura y su entorno. El modo de hacerlo es mediante proyectos colaborativos de base local. Ha trabajado con las Naciones Unidas, por ejemplo, en sistemas alimentarios; en apoyo en situaciones de desastre como las acontecidas en Nepal en 2015, entre otros. Algo menos de un centenar de organizaciones, fundaciones particulares, ministerios, departamentos gubernamentales, universidades privadas dan soporte a esta organización ${ }^{88}$.

\section{The International Mountain Society (IMS)}

Esta sociedad fue fundada en 1980 a raíz de la atención que se dio al estudio de las montañas desde el programa $M A B$. La Universidad de las Naciones Unidas (UNU) acogió la iniciativa de su creación (Ives, 1981). Esta asociación tiene su sede en Berna (Suiza). Sus objetivos son el de promover las investigaciones sobre el medio de montaña y su divulgación. Su órgano de difusión es la revista Mountain Research and Development. La IMS, junto con otras organizaciones no gubernamentales, tuvo un papel fundamental en la elaboración del capítulo 13 de la Agenda 21, conocida también como Agenda de montaña 1992 (Mountain Agenda 1992) ${ }^{89}$. Asimismo, ha contribuido a crear asociaciones regionales para la investigación de las zonas de montaña en diversos países ${ }^{90}$. La sociedad está financiada actualmente por tres instituciones, Austrian Development Agency (ADA), el Centre for Development and Environment, de la Universidad de Berna (Suiza) (que acoge la Mountain Research Initiative, MRI), y el International Centre for Integrated Mountain Development (ICIMOD) sito en Katmandú, Nepal.

\section{The Mountain Forum}

El origen y desarrollo de esta red sobre temas de montaña está explicada por la primera de sus responsables Elizabeth Byers (1998). En los años setenta se organizó un grupo de investigadores que trabajaban en temas de montaña. Este grupo fue posteriormente clave en la constitución de la

\footnotetext{
$87<$ https://mountain.org>.

${ }^{88}$ Pratt, 2000.

${ }^{89}$ CSD, 1995, pár. 43 y 82.

${ }^{90}$ Ibidem, pár. 68.
} 
Agenda de montaña (Mountain Agenda) ${ }^{91}$ al que se adhirieron otras organizaciones que impulsaron la inclusión de un capítulo dedicado a la montaña. La formalización de Mountain Forum fue a partir de 1995 aprovechando las crecientes posibilidades de interconexión de la World Wide Web. En 1997 se constituyó como tal y se alojó en The Mountain Institute, en West Virginia (EE.UU.). Su finalidad era la del intercambio de ideas y experiencias en torno a la montaña. Al mismo tiempo se crearon dos nodos regionales, uno en Asia, la Asian Pacific Mountain Network, dentro de ICIMOD, en Kathmandú (Nepal), y otro en Ecuador, a través de Infoandina, en el Centro Internacional de la Papa (CIP) (International Potato Center).

Mountain Forum ha organizado conferencias y ha participado en la organización de eventos de alcance internacional relativos al ámbito de la montaña. También cuenta con una biblioteca virtual de gran interés. Originalmente toda la comunicación se hacía vía correo electrónico, pero hoy dispone de otras vías de comunicación a partir de redes sociales como son Facebook o Tweeter.

\section{Mountain Research Initiative ${ }^{92}$}

Si, como hemos mencionado, en 1992 la Agenda 21 recogía la preocupación por el devenir de las montañas en el mundo, se puede decir que a partir de ella se generaron diversas iniciativas, se crearon organismos y se promovieron programas, tendientes a conocer mejor desde el ámbito internacional, las montañas: el medio físico y biológico, y la acción desarrollada por el ser humano. Uno de los resultados fue Mountain Research Initiative (MRI) que se presenta como una red internacional, una comunidad, interesada en "generar conocimientos que permitan adoptar decisiones, acciones, y transformaciones hacia el desarrollo sostenible" en los ambientes de montaña del mundo, principalmente afectados por el cambio global. La Coordinación de MRI se aloja en el Centre for Development and Environment de la Universidad de Berna (Suiza). Sirve como punto de conexión $(h u b)$ de toda la información generada sobre las diferentes áreas montañosas del mundo. Inicialmente acogía diversos proyectos como la International GeosphereBiosphere Programme (IGBP), la International Human Dimensions Programme (IHDP) y el Global terrestrial Observing Sysemm (GTOS).

Consorcio para el Desarrollo Sostenible en los Andes, CONDESAN ${ }^{93}$

CONDESAN es una organización fundada en 1993, originariamente para asesorar como parte del Grupo Consultivo para la Investigación agrícola internacional (CGIAR) y desde 2009 como organización regional autónoma con la intención de proveer un enfoque ecosistémico a los proyectos de desarrollo que se lleven a cabo en las regiones andinas. Su trabajo es a nivel local y trata de vincular agencias gubernamentales estatales con organizaciones no gubernamentales (ONG). Actualmente CONDESAN lleva la Secretaría Técnica de la Iniciativa Andina de Montaña.

\footnotetext{
${ }^{91}$ Mountain Agenda, 2001.

$92<$ https://www.mountainresearchinitiative.org >.

$93<$ https://condesan.org $>$.
} 
Las noticias relacionadas con el desarrollo sostenible en las montañas andinas pueden consultarse en Infoandina ${ }^{94}$, organización vinculada también al proyecto CONDESAN. Infoandina nos remite a numerosas publicaciones, foros, instituciones, entre otras, ligadas al mundo de la montaña.

\section{International Centre for Integrated Mountain Development, ICIMOD ${ }^{95}$}

Es una organización intergubernamental creada en 1983 con centro en Kathmandú (Nepal) con el ánimo de impulsar el desarrollo económico y ambiental, y el bienestar en las comunidades de esta gran cadena montañosa de Hindu-Kush-Himalaya. Promueve la investigación aplicada, y el acopio de experiencias, de documentación sobre proyectos realizados en el conjunto de la región, aparte de formar a especialistas en diferentes áreas siempre relacionados con proyectos de desarrollo local en cualquiera de los ocho países que forman parte de él, a saber: Afghanistan, Bangladesh, Bhutan, China, India, Myanmar, Nepal y Pakistán. Es también una plataforma de encuentro entre científicos, políticos y la sociedad.

ICIMOD ofrece programas regionales, proyectos de adaptación y resiliencia para comunidades, proyectos integrales de gestión de cuencas transnacionales, entre otros muchos más. ICIMOD es financiado por los gobiernos de los diferentes países de la región asociados, además de los gobiernos de países como los escandinavos, Austria, Suiza y Australia y de instituciones de diversos países.

\section{Comisión Internacional para la Protección de los Alpes, CIPRA 96}

Es la Comisión Internacional para la Protección de los Alpes. Esta organización abarca regiones de siete países, a saber, Austria, Alemania, Eslovenia, Francia, Italia (sud Tirol y Trieste), Liechtenstein, Suiza.

Fue fundada en 1952 por iniciativa de la Unión Internacional para la Conservación de la Naturaleza (IUCN) con el ánimo inicial de proteger los espacios naturales bajo una óptica transfronteriza. Posteriormente, tras la crisis de la organización en los años setenta se abre la posibilidad de ampliar los temas de interés entre las comunidades alpinas para dar cobertura unitaria a las poblaciones que viven en esta región multicultural geográfica. Posteriormente, con la aprobación de la Convención de los Alpes por parte de la Unión Europea en 1991, forma parte de los órganos consultivos ${ }^{97}$. Con este Convenio se trataba de dar un tratamiento común en el marco de la Unión Europea al conjunto de los espacios naturales, de la gestión de sus recursos, del turismo, de los proyectos energéticos, de solución de los problemas locales, entre otros.

\section{Publicaciones de interés por las montañas}

Independientemente de que, en algunas fechas relevantes recientes para el mundo de la montaña, las revistas de Geografía han dedicado algún ejemplar a su tratamiento, por ejemplo, GeoJournal, Geographical Review, en 2002, o los Annales de la Asociación de Geógrafos Americanos en

$94<$ http://infoandina.org/infoandina/>

${ }^{95}<$ https://www.icimod.org >. Véase también ICIMOD, 1981.

$96<$ https://www.cipra.org $>$.

${ }^{97}$ Decisión 96/191/CE del Consejo europeo, aunque entró en vigor el 4 de abril de 1998. 
La montaña em los programas internacionales de la UNESCO (1972-2002): del Man and Biosphere...

$2017^{98}$, existen una serie de publicaciones periódicas que vale la pena revisar para entender el tipo de investigaciones que se han realizado y las que se están haciendo sobre este medio. Entre ellas:

\section{Revue de Géographie Alpine/ Journal of Alpine Research}

Es la revista del Instituto de Geografía Alpina de la Universidad de Grenoble. Es por supuesto la institución emblemática en el estudio de la montaña desde su creación por Raoul Blanchard en la segunda década del siglo pasado (1913). Originalmente el título de la publicación era Recueil des Travaux de l'Institut de Géographie Alpine, hasta 1919 en que adoptó el nombre actual. Desde 2004, la Revue comenzó a publicarse en inglés con el título Journal of Alpine research. Admite una amplia variedad de enfoques y temáticas mayoritariamente relacionados con el mundo alpino.

\section{Arctic, Antarctic and Alpine Research}

Publicación de la Universidad de Colorado, su nombre original era Arctic and Alpine research (1969-1998), vinculada a un instituto de investigación que con el mismo nombre había dentro de esta universidad. Desde sus inicios en 1969 se planteó una línea editorial interdisciplinaria de manera que pudiera acoger artículos procedentes de especialistas de las ciencias sociales, siempre ligadas con los dos temas centrales de la revista. Su primer director fue Jack D. Ives. Desde 1998 cambió su nombre para incluir los ecosistemas antárticos, de ahí su título actual.

\section{Mountain Research and Development ${ }^{99}$}

Vigente desde 1981 hasta la actualidad, es el órgano difusor de la International Mountain Society. Desde sus orígenes su intención es la de albergar todas las investigaciones relacionadas con la montaña, con un enfoque interdisciplinario. Se puede consultar su contenido gratuitamente en su portal para los ejemplares desde 2010 hasta la fecha.

\section{Pirineos. Revista del Instituto Pirenaico de Ecología 100}

Fundada en 1945 como órgano difusor del Instituto de Estudios Pirenaicos dentro del Consejo Superior de Investigaciones Científicas, en España. Fue precedida por un boletín que recogía las investigaciones que se realizaban en la entonces llamada Estación de Estudios Pirenaicos. Tras un período de escasa actividad la publicación fue relanzada en 1987 para acoger los estudios ecológicos de las regiones de montaña, no solamente de los Pirineos, lo cual abrió el panorama y la revitalizó. Actualmente es una de las revistas enseña, a nivel internacional, sobre estudios de la montaña.

\footnotetext{
${ }^{98}$ La Asociación norteamericana dedicó un número especial en 2017 a los temas de montaña pero previamente ya había constituido una sección encabezada y fundada en 1999 por Donald A. Friend bajo el título de Geografía de la montaña (Mountain Geography).

${ }^{99}$ Esta revista es de libre acceso a partir de los ejemplares de 2010 en adelante <https://www.mrd-journal.org >. Desde el portal BioOne se pueden consultar los números desde el año $2000<\mathrm{https}$ ///bioone.org/journals/mountainresearch-and-development>. Finalmente, a los números anteriores, desde 1981, se puede acceder a través de Jstor <https://www.jstor.org/journal/mounresedeve $>$.

$100<$ http://pirineos.revistas.csic.es/index.php/pirineos $>$.
} 
Journal of Mountain Science $e^{101}$

Producto de la Academia de Ciencias China inició su singladura en 2004. Está financiada por Chengdu Institute of Mountain Hazards and Environment y cuenta con el apoyo de la Universidad de las Naciones Unidas (UNU).

\section{Journal of Mountain Research ${ }^{102}$}

De la Society for Himalayan Action Research and Development (SHARAD), la H.N.B. Garwhal University y otras instituciones, se publica desde 2006. Sus temas prioritarios son investigaciones del medio físico y biológico de las regiones de montaña.

\section{Bibliografía}

ALLAN, Nigel J. R. Mountain Environments. GeoJournal. Vol. 27. Núm. 1. Mayo 1992. p. 5-11. <https://link.springer.com/article/10.1007/BF00150631> [5 de agosto de 2019].

ALLAN, Nigel J. R. Ratzel revisited. Human aspects of mountain environmental change, 18891992. Mohammad Shafi, Mehdi Razh. Geography of Environment. Jaipur \& New Delhi: Rawat Publications, 1994. [s/p] <https://archive.org/stream/in.ernet.dli.2015.131774/2015.131774.IndiaAnd-The-World_djvu.txt>.

ALLAN, Nigel J. R.; KNAPP, Gregory W.; STADEL, Christoph (Eds.). Human Impact on Mountains. Lanham, Maryland: Rowman and Littlefield, 1988, 308 p.

BENISTON, Martin. Environmental Change in Mountain and Uplands. Londres/ Nueva York. Routledge, 2016, 188 p.

BHATT, Dibya Deo. Regional Meeting on Integrated Ecological Research and Training Needs in the southern Asian Mountain Systems. Particularly the Hindu-Kush-Himalayas. Final Report Man and Biospehere Report Issues. Vol. 3. Issue 3. Autumn, 1976. p. 232. <https://unesdoc.unesco.org/ark:/48223/pf0000017776?posInSet=1\&queryId=8c96fc0d-8d384c7b-9eef-254ed5832765> [2 de febrero de 2020].

BISHOP, M. P. International Multidisciplinary Research and Education: A Mountain Geography Perspective. Geography, Geographers and Study Abroad. Journal of Geography. Vol. 109. Núm. 3. 2009. p. 112-120.

BLACHE, Jules. L’homme et la montagne. Prólogo de R. Blanchard. Paris: Gallimard, 1934,192p.

BLANCHARD, Raoul. Reseña: De Martonne (Emmanuel). Les Alpes. Géographie générale. Revue de Géographie Alpine. Vol. 15, Núm .1. 1927. p. 166-168.

$101<$ http://jms.imde.ac.cn/jms>.

$102<$ http://jmr.sharadpauri.org $>$. 
La montaña em los programas internacionales de la UNESCO (1972-2002): del Man and Biosphere...

BLANCHARD, Raoul. Reseña: Ramond. Voyage dans les Pyrenées, précédé de la Jeunesse de Ramond par André Mongland. Revue de Géographie Alpine. Vol. 15, Núm. 2. 1927. p. 370-372.

BLANCHARD, Raoul. Les Alpes occidentales. Grenoble: Arthaud, 1937-1956, 7 Tomos.

BLYTH, Simon (Eds.). Mountain Watch: Environmental Change and Sustainable Development. United Nations Environment Programme (UNEP)/Earthpoit, 2002, 80 p. <https://www.unepwcmc.org/resources-and-data/mountain-watch--environmental-change-sustainable-developmentin-mountains> [6 de abril de 2020].

BUTLER, David R. Roderick Peattie's Mountain Geography-A critique and field study. Progress in Physical Geography. 2017. Vol 41. Núm. 2. p. 238-242.

BYERS, Elizabeth A. The Mountain Forum. Networking for Mountain Conservation and Development. Unasylva. An International Journal of Forestry and forest industries. Vol. 49, Moving Mountains. $\mathrm{n}^{\circ} 195$. 1998/4 s/p. <www.fao.org/3/w9300e/w9300e04.htm> [22 de febrero de 2020].

CES (Consejo Económico y Social). Resolutions and Decisions of the Economic and Social Council (E/1998/98). Nueva York, $2000, \quad$ p. 61. <https://www.un.org/en/development/desa/policy/cdp/cdp_res_dec/e_1998_46.pdf>[5 de abril de 2020].

CSD (Comisión para el Desarrollo Sostenible). "Review of Sectorial Clusters, second phase: Land, Desertification, Forests and Biodiversity. Managing fragile ecosystems: sustainable mountain development". Report of the Secretary-General. E/CN.17/1995/10 de febrero de 1995. Nueva York: Consejo Económico y Social de las Naciones Unidas. Tercera Sesión. 11 a 28 de abril de 1995. <https://sustainabledevelopment.un.org/index.php?menu=1174> [25 de febrero de 2020].

CSD (Comisión para el desarrollo Sostenible). Integrated Planning and Management of Land Resources. Report of the Secretary-General. Addendum. E/CN.17/2000/6/Add.3. Sustainable Mountain Development. Economic and Social Council (United Nations), 1 de febrero de 2000. <https://www.un.org/esa/documents/ecosoc/cn17/2000/ecn172000-6add2.htm> [10 de febrero de 2020].

DASMANN, Raymond Frederic; POORE, Duncan. Ecological Guidelines for Balanced Use, Conservation and Development in High Mountains. Gland (Switzerland): Internationa Union for Conservation of Nature and Natural Resources (IUCN)/ United Nations Environemnt Programme (UNEP)/ World Wildlife Fund (WWF), $1979, \quad 40 \quad$ p. $<$ https://books.google.com.mx/books?id=HIPwAAAAMAAJ\&printsec=frontcover\&hl=es\#v=tw opage \&q\&f=false> [7 de febrero de 2020].

DENNISTON, Dereck. Máxima prioridad. La conservación de los ecosistemas y cultura de montaña. Bilbao: Bakeaz, 1996, 
DIVISIÓN PARA EL DESARROLLO SOSTENIBLE (Departamento de las Naciones Unidas para Asuntos Económicos). Mountains. 2010-2011 CSD Topics (Comisión para el Desarrollo Sostenible).

<https://sustainabledevelopment.un.org/content/dsd/susdevtopics/sdt_mountains.shtml>. [14 de febrero de 2020].

ECKHOLM, Eric P. Losing ground. Environmental stress and world food prospect. New York: Norton, 1976, 223 p.

ECKHOLM, Erik P. The deterioration of Mountain Environments. Science. Vol. 189. Núm. 4205. 5 de septiembre de 1975. p. 764-770. <https://science.sciencemag.org/content/189/4205/764> [5 de agosto de 2019].

ENVIRONMENTAL CONSERVATION. Notice: The State of the World's Mountains: A Global Report. Environmental Conservation. Vol. 20. Núm 1. $1993 . \quad$ p. 96. $<$ https://www.cambridge.org/core/journals/environmental-conservation/article/notice-the-stateof-the-worlds-mountains-a-global-report-edited-by-stone-peter-

b/A17B6496EA155AF5770F4FF75E0B5060> [22 de febrero de 2020].

FRIEND, Donald. Formation of a Mountain Geography Specialty Group within the Association of American Geographers (AAG). Mountain Research and Development. Vol. 19. Núm. 2. 1999. p. 167-168

FUNNELL, Don; PARISH, Romola. Mountain Environments and Communities. London: Routledge, 2005, 432 p. <https://bidi.uam.mx:6990/10.4324/9780203992067> [9 de abril 2020].

FUNNELL, Don; PRICE, Martin F. Mountain Geography: a Review. The Geographical Journal. Vol. 169. Núm. 3. September, 2003. p. 183-190.

GERRARD, John. Mountain Environments: an Examination of the Physical Geography of Mountains. Cambridge: Massachussets Institute of Technology, 1990, 317 p.

GONZÁLEZ TRUEBA, Juan José. Carl Troll y la geografía del paisaje: vida, obra y traducción de un texto fundamental. Boletín de la Asociación Española de Geógrafos. Núm. 59. 2012. p. 173200. <https://bage.age-geografia.es/ojs/index.php/bage/article/view/1454/1375> [5 de agosto de 2019].

HEWITT, Kenneth. The Study of Mountain Lands and Peoples. In: Nigel J. R. Allan; Gregory W. Kanpp; Christoph Stadel (Eds.). Human Impact on Mountains. Boston: Rowman and Littkefield Publishers, Inc., 1988, p. 6-23.

ICIMOD: A New Centre for South-Central Asian Mountain Development. Mountain Research and Development. Vol. 3. Núm. 2. Workshop on the Stability and Unstability of Mountain Ecosystems. Berne-Riederalp. 14-19 de septiembre de 1981 ( $1^{\text {a }}$ parte). Mayo de 1983, p. 186. 
La montaña em los programas internacionales de la UNESCO (1972-2002): del Man and Biosphere...

INTERNATIONAL MOUNTAIN SOCIETY. Mountain Ecosystems: Stability and Instability. Proceedings of a Workshot. Berne-Riederalp, Switzerland, 14- 19 September, 1982. Mountain Research and Development. Boluder (Clorado). Vol. 3, Núm.2. May. 1983.

IVES, Jack D. International Mountain Society. Crisis in the Mountains. Mountain Research and Development. Vol.1. Núm. 2. 1981. p. 188-191.

IVES, Jack D. Regional Meeting on Integrated Ecological Research and Training Needs in the southern Asian Mountain Systems. Particularly the Hindu-Kush-Himalayas. Final Report Man and Biospehere Report Issues, Núm. 34. Paris. 1977.

IVES, Jack. The Theory of Himalayan Degradation. Its Validity and application Challenge by Recent Research. Mountain Research and Development. Vol. 7. Núm. 3. Proceedings of the Mohonk Mountain Conference: The Himalaya-Ganges Problem. Agosto, 1987. p. 189-199.

IVES, Jack D. The Origins of Mountain Geoecology. Pirineos. Revista de ecología de montaña, 2012, Vol. 167. p. 15-27.

IVES, Jack D. Himalayan perceptions: environmental change and the Well-Being of Mountain Peoples. Lalitpur: Himalayan Association for the Advancement of Sciences, 2006, 274 p. [1 ${ }^{\text {a }}$ edición Londres/Nueva York: Routledge, 2004] <https://bidi.uam.mx:6990/10.4324/9780203597569> [9 de abril 2020].

IVES, Jack D. MESSERLI, Bruno. The Himalayan Dilemma: Reconciling Development and Conservation. London: Routledge/United Nations University (UNU), 1989, 295 p.

IVES, Jack D.; MESSERLI, Bruno. Stability and Unstability on Mountain Ecosystems. Mountain Research and Development. Vol. 4. Núm. 1. 1984. p. 63-71.

IVES, Jack D.; MESSERLI, Bruno. Mountain Geoecology. The evolution of intellectually-based scholarship into a political force for Sustainable mountain development. In: Maria Sala (Ed.). UNESCO. Encyclopedia of Life Support System (EOLSS). 2009. Vol. I, No 14. p 344- 363. <https://www.eolss.net/Sample-Chapters/C01/E6-14-02-08.pdf> [11 de noviembre de 2019].

KAPOOR, Anup; KAPOOR, Satwanti. Ecology and Man in the Himalayas. New Delhi: M.D. Publications, 1994.

KAPOOR, Anup. K.; SINGH, Dharamvir. Man and Development in the Himalayas. Delhi: Academic Foundation, 1996, 241 p.

KREUTZMANN, Hermann. From Water Towers of Mankind to Livelihood Strategies of Mountain Dwellers. Erdkunde. Vol. 52. Núm. 3. Julio- septiembre, 1998. p. 185-200.

LALL, J. S.; MODDIE, A. D. (Eds.) Himalaya. Aspects of Change. Nueva Delhi: India International Centre. Oxford University 1981. 
<http://14.139.206.50:8080/jspui/bitstream/1/4151/1/The\%20Himalaya\%20Aspects\%20of\%20C hange_2.pdf> [25 de Agosto de 2019].

MEADOWS, Dennis; MEADOWS, Donella; RANDERS, Jörgen; BEHRENS Wlliam W. III. The Limits to Growth: A Report for the Club of Rome's Project on the Predicament of Mankind. New York: Universe Books, 1972, 205 p.

MESSERLI, Bruno; IVES, Jack D. Mountains of the World: A Global Priority. London/ New York: The Parthenon Publishing Group, 1997, 495 p.

MEYZENQ Claude. Géographie de la montagne (Mountains geography). Bulletin de l'Association de géographes français, 73e année, 1996-3 (juin). La recherche géographique en France. p. 240242.

MILLER, R. Gordon. Review: The State of the World's Mountains. A Global Report. Edited by P. B. Stone. Environmental Conservation. Vol. 21. Núm 1. 1994. p. 88.

MOUNTAIN AGENDA. The Mountain Agenda. Mountain Research and Development. Vol. 20. Núm. 3. 2001. p. 286.

MOUNTAIN PARTNERSHIP. International Year of Mountain Documents. $<$ http://www.fao.org/mountain-partnership/publications/policybriefs/international-year-ofmountains-documents/en/> [14 de febrero de 2020].

MOUNTAIN RESEARCH and Development. Mountains of the World: a Global Priority (Review). Mountain Research and Development vol. 17. Núm. 4. Noviembre, 1997. p. 375-376.

NEUSTADTL, S. Montology: the Ecology of Mountains. Technological Review. Vol. 79. Núm. 8. p. 64-66.

NICHOLS, Sandra; MON PERE, Carol. The fragile mountain. The Sandra Nichols Productions. $<$ https://archive.org/details/thefragilemountain/thefragilemountainreel1.mov> [1 de septiembre de 2019].

ONU (Organización de las Naciones Unidas). Agenda 21. Departamento de Asuntos Económicos y Sociales. División de Desarrollo Sostenible. Diciembre de 1992a. <https://www.un.org/spanish/esa/sustdev/agenda21/index.htm> [22 de agosto de 2019]. También puede consultarse en $\langle$ https://sustainabledevelopment.un.org/content/documents/Agenda21.pdf >.

ONU (Organización de las Naciones Unidas). Informe de la Conferencia de las Naciones Unidas sobre el Desarrollo Sostenible. Río de Janeiro (Brasil), 20 a 22 de junio de 2012. (A/CONF.216/16). Nuevas York: Naciones 2012 $<$ https://sustainabledevelopment.un.org/index.php?page=view \&type=111\&nr=1358\&menu=35> [5 de abril de 2020]. 
La montaña em los programas internacionales de la UNESCO (1972-2002): del Man and Biosphere...

ONU. Informe de la Secretaría General. Special edition: progress towards the Sustainable Goals. Consejo Económico y social. E/2019/68. 8 de mayo de 2019. <https://undocs.org/E/2019/68> [5 de abril de 2020].

ONU. Resolution adopted by the General Assembly. 53/24 International Year of Mountains. A/RES/53/24 de 19 de noviembre de 1998. <https://undocs.org/en/A/RES/53/24> [20 de febrero de 2020].

ONU (Organización de las Naciones Unidas). Día Internacional de las montañas. 11 de diciembre. 2019. <https://www.un.org/es/observances/mountain-day> [10 de febrero de 2020].

PEATTIE, Roderick. Mountain Geography. A Critique and Field Study. Cambridge, Massachussets: Harvard University Press, 1936, 257 p.

PRATT, Jane. The Mountain Institute. Mountain Research and Development. Vol. 20. Núm. 3. Agosto. 2000. p. 280-281.

PRICE, Larry W. Mountain and Man. A Study of Process and Environment. Berkeley: University of California, 1981, $527 \mathrm{p}$.

PRICE, Martin F. Mountains: globally important ecosystems. Unasylva. An International Journal of Forestry and Forest Industries Vol. 49. Moving mountains. Núm. 195. 1998/4. s/p. <www.fao.org/3/w9300e/w9300e03.htm> [22 de febrero de 2020].

PRICE, Martin F.; BYERS, Alton C. et al. (Eds.) Mountain Geography: Physical and Human Dimensions. Berkeley: University of California Press, 2013, 400 p.

PRICE, Martin F.; JANSKY, Libor F.; IATSENIE Andrei A. (Eds.) Key Issues for Mountain Areas. Tokyo/ New York/ Paris: United Nations University Press, 2004, 273 p.

RATZEL, Friedrich. Kleine Schriften von Friedrich Ratzel Bd. Beiträge zur physyschen Erdkunde, Ethnographie und Anthropogeographie. Anhang Ratzel Bibliographie 1867- 1908. München und Berlin: Druck und Berlag von R. Oldenbourg, 1908. <https://archive.org/stream/bub_gb_egNbAAAAQAAJ/bub_gb_egNbAAAAQAAJ_djvu.txt> [6 de febrero de 2020].

RIEGER, Hans Ch., BIERI, F.; EGGERS, H.; GOLDSCHALT, W. ; STEIGER, J. Nepal Report. Heidelberg: Himalayan Ecosystems Research Mission, 1976a.

RIEGER, Hans Ch., BIERI, F.; EGGERS, H.; GOLDSCHALT, W. ; STEIGER, J. India Report. Heidelberg: Himalayan Ecosystems Research Mission, 1976 b.

SARMIENTO, Fausto. Breaking Mountain paradigm: Ecological Effect on Human Impact in Managed Tropandean Landscapes. Ambio. Vol. 29. Núm. 7. 2000. p. 423-431. 
SARMIENTO, Fausto. Obituary for a Mountain man. Mountain Research and Development. Vol. 30. Núm. 2. 2010. p. 188.

SARMIENTO, Fausto. The Montology Manifiesto: Echoes Towards a Transdisciplnary Science of Mountains. Journal of Mountain Science. En publicación.

SETHI, Sunil. Problems of deforestation and soil erosion in Himalayas. India today. May 15.1976 [Actualizado en april 2015]. <https://www.indiatoday.in/magazine/environment/story/19760515-problems-of-deforestationand-soil-erosion-in-himalayas-819149-2015-04-04> [27 de febrero de 2020].

SHAFI, Mohammad; RAZH, Mehdi. Geography and Environment. Jaipur y New Delhi: Rawat Publications, 1994. <https://archive.org/stream/in.ernet.dli.2015.131774/2015.131774.India-AndThe-World_djvu.txt> [5 de febrero, 2020].

SINGH, Dynamics of Mountain. Geo-Systems. New Delhi: Ashish Publishing Houses, 1992.

SMETHURST, David. Mountain Geography. Geographical Review, vol. 90(1). Enero, 2000. p. $35-56$

SMITH, Guy Harold. Roderick Peattie, a Geographer and Romanticist, 1891- 1955. Annals of the Association of American Geographers. 1957. Vol. 47. Núm. 1. p. 96-99.

SOFFER, A. Mountain Geography: a New Approach. Mountain Research and Development. Vol. 2. Núm. 4. 1982. p. 391-398.

STADEL, C. Mountain Regions. Their Nature and Problems. Geographical perspectives. Núm. 49. 1982. p. 26-33.

STONE, Peter Bannet. (Eds.). The State of the World's Mountains: A Global Report. London: Zeed Books, 1994, XX, 391 p.

SUNYER MARTÍN, Pere; MONTERROSO SALVATIERRA, Neptalí. Los espacios de montaña en México. Del control comunitario al (des)control neoliberal. Scripta Nova. Revista electronica de Geografía y Ciencias Sociales. Universidad de Barcelona. Vol. XVIII. Núm. 493(57), 1 de noviembre de 2014. <https://revistes.ub.edu/index.php/ScriptaNova/article/view/15046/18399>.

THORSELL, Jim, HAMILTON, Larry. A Contribution to the Global Theme Study of World Heritage Natural Sites. Working Paper 6. International Union for Conservation of Nature (UICN). United Nations, September, 2002

THORSELL, Jim; HAMILTON, Lawrence. A Global Overview of Mountain Protected Areas on the World Heritage List: a Contribution to the Global Theme Study of World Heritage Sites. IUCN, World Commission on Protected Areas, Banff Centre (Canada), 2002, 34 p. 
La montaña em los programas internacionales de la UNESCO (1972-2002): del Man and Biosphere...

TOBIAS, Michael C.; DRASDO, Harold. The Mountain Spirit. New York: Woodstock, 1979, 264p.

TOBIAS, Michael C. Mountain People. Norman \& London: University of Oklahoma Press, 1986.

TROLL, Carl. Geo-ecology of the Mountainous Regions of the Tropical Americas. Proceedings of the UNESCO Mexican Symposium. 1 al 3 de agosto, 1966. Bonn: Dümmler in Kommission, 1968, $223 \mathrm{p}$.

TROLL, Carl. Comparative Geography of High Mountains of the World in the View of Landscape Ecology: A Development of Three and a Half Decades of Research an Organization. In: Nigel J. R. Allan; Gregory W. Knapp; Christoph Stadel (Eds.). Human Impact on Mountains. Lanham, Maryland: Rowman and Littlefield, [1975] 1988, p. 36- 56.

TROLL, Carl. Landschaftsökologie. Reinhold Tüxen. Pflanzengeographie und Landscahftsökologie. Bericht über das 7. Internationales Symposion in Stolzenau/Weser, 1963 der Internationales Vereinigung für Vegetationskunde. The Hague: Junk N. V. Publishers, 1968, p. 121.

TÜXEN, Reinhold. Pflanzensoziologie und Lansdchaftsökologie. Bericht über das 7. Internationales Symposion in Stolzenau/Weser, 1963 der Internationales Vereinigung für Vegetationskunde. The Hague: Junk N. V. Publishers, 1968, 426 p.

UGI (Unión Geográfica Internacional). Comisión de Geoecología de montaña. Commission on Mountains Geoecology. Symposium in Japan Alps. August, 27- 31, 1980. Mountain Research and Development. Vol. 1. Núm. 1. 1981. p. 83-84.

UGI. Geoecology of the high-mountain regions of Eurasia: Proceedings of the International Geographical Union Commission on High-altitude Geoecology. Erdwissenschaftliche Forschung. Symposium of the International Geographical Union Commission on High-altitude Geoecology, Mainz (Alemania), 20-22 November 1969. Wiesbaden: Franz Steiner, 1972. xvii, 299p., xxv: ill., maps.

UGI. International Geographical Union Study Group. Commission on Diversity in Mountain Systems C04.08. Newsletter. <http://www.homeofgeography.org/uk/e-Arch_1/C04.08.html>.

UNESCO. Projet du programme intergouvernemental et interdisciplinaire à long terme sur l'homme et la biosphère. Conférence Générale. Seizième session. Paris 1970. 16C/78. 6 de octubre de 1970. <https://unesdoc.unesco.org/ark:/48223/pf0000161015_fre> [6 de agosto de 2019].

UNESCO. Expert Panel on Project 6. Impact of the Human Activities on Mountain Ecosystems. Salzburg (Austria), 29 de enero al 4 de febrero de 1973. Final Report. (SC.73/CONF.615/2). 69 p. 1973a. <https://unesdoc.unesco.org/ark:/48223/pf0000004980> [6 de agosto de 2019]. 
UNESCO. International Co-ordinating Council of the Program on Man and the Biosphere. First session. Paris, 9- 19 of November, 1971. Final Report. Report Series. Núm. 1. SC/MD/26. Paris, 25 February, 1972. <https://unesdoc.unesco.org/ark:/48223/pf0000004354> [6 de agosto, 2019].

UNESCO. Working Group on Project 6. Impact of Human Activities on Mountain and Tundra Ecosystems. Lilehammer (Norway), 20 al 23 de noviembre de 1973 (SC.73/CONF.656/1), 132 p. 1974. <https://unesdoc.unesco.org/ark:/48223/pf0000009336> [6 de agosto de 2019].

UNESCO. Regional Meeting on Integrated Ecological Research and Training Needs in the Andean Region. La Paz (Bolivia). 10 a 15 de junio de 1974. Final Report. (SC.74/CONF.694/1) 61 p. 1975a. <https://unesdoc.unesco.org/ark:/48223/pf0000016231> [3 de septiembre de 2019].

UNESCO. Regional Meeting on Integrated Ecological Research and Training Needs in the Southern Asian Mountain Systems, particularly the Hindu-Kush Himalaya. Kathmandú (Nepal). 26 de septiembre a 2 de octubre de 1975. Final Report. 75 p. 1975b. https://unesdoc.unesco.org/ark:/48223/pf0000025632?posInSet=10\&queryId=e36f162e-23014ebe-972b-7221644f5886> [3 de septiembre de 2019]. Resumen en <https://unesdoc.unesco.org/ark:/48223/pf0000017776?posInSet=1\&queryId=8c96fc0d-8d384c7b-9eef-254ed5832765>.

UNESCO. Reunión Regional de planificación de los comités nacionales andinos del MAB con especial énfasis en el Proyecto 6, Lima 1975: Informe final. Lima, 2 a 5 de Diciembre de 1975. Serie de Informes del MAB n³9. 1976. <https://unesdoc.unesco.org/ark:/48223/pf0000027509_spa?posInSet=1\&queryId=765204be4be3-4668-8d2b-9451a63ece0f> [2 de febrero de 2020].

VANCE, Rupert B. Reviewed work: Mountain geography: A Critique and Field Study. American Sociological Review. Vol. 4. Núm. 5. Octubre, 1939. p. 751-752.

(C) Copyright: Pere Sunyer Martín, 2020.

(C) Copyright:Ar@cne, 2020.

Ficha bibliográfica:

SUNYER MARTÍN, Pere. La montaña en los programas internacionales de la UNESCO (1972-2002): del Man and Biosphere al Año Internacional de las Montañas. Fuentes para su investigación. Ar@cne. Revista Electrónica de Recursos de Internet sobre Geografía y Ciencias Sociales. Barcelona: Universidad de Barcelona, 1 de agosto de 2020, vol. XXIV, no 245 <DOI: https://doi.org/10.1344/ara2020.245.32115>.

\section{Menú principal de Geo Crítica}

